\title{
MICROFLUIDIC ELECTRICAL IMPEDANCE SPECTROSCOPY
}

\author{
A Thesis \\ presented to \\ the Faculty of California Polytechnic State University, \\ San Luis Obispo
}

\author{
In Partial Fulfillment \\ of the Requirements for the Degree \\ Master of Science in Biomedical Engineering
}

by

John J. Foley

September 2018 
(C) 2018

John J. Foley

\section{ALL RIGHTS RESERVED}


COMMITTEE MEMBERSHIP

TITLE: Microfluidic Electrical Impedance Spectroscopy

AUTHOR: John J. Foley

DATE SUBMITTED: September 2018

COMMITTEE CHAIR: Benjamin Hawkins, Ph.D. Assistant Professor of Biomedical Engineering

COMMITTEE MEMBER: David Clague, Ph.D.

Professor of Biomedical Engineering

COMMITTEE MEMBER: Christopher Heylman, Ph.D.

Assistant Professor of Biomedical Engineering 


\begin{abstract}
Microfluidic Electrical Impedance Spectroscopy

John J. Foley
\end{abstract}

The goal of this study is to design and manufacture a microfluidic device capable of measuring changes in impedancevalues of microfluidic cell cultures. Tocharacterize this, an interdigitated array of electrodes was patterned over glass, where it was then bonded to a series of fluidic networks created in PDMS via soft lithography. The device measured ethanol impedance initially to show that values remain consistent over time. Impedance values of water and $1 \% \mathrm{wt}$. saltwater were compared to show that the device is able to detect changes in impedance, with up to a $60 \%$ reduction in electrical impedance in saltwater. Cells were introduced into the device, where changes in impedance were seen across multiple frequencies, indicating that the device is capable of detecting the presence of biologic elements within a system. Cell measurements were performed using $\mathrm{NIH}-3 \mathrm{~T} 3$ fibroblasts. 


\section{ACKNOWLEDGMENTS}

Special thanks to:

- Benjamin Hawkins: For his hard work, dedication, time, and guidance

- David Lange: For his assistance in manufacturing processes and LabView code

- Kevin Wolfarth: For his assistance in microscopy and gradient validation

- My family and friends: For always being there as my support and counsel 


\section{TABLE OF CONTENTS}

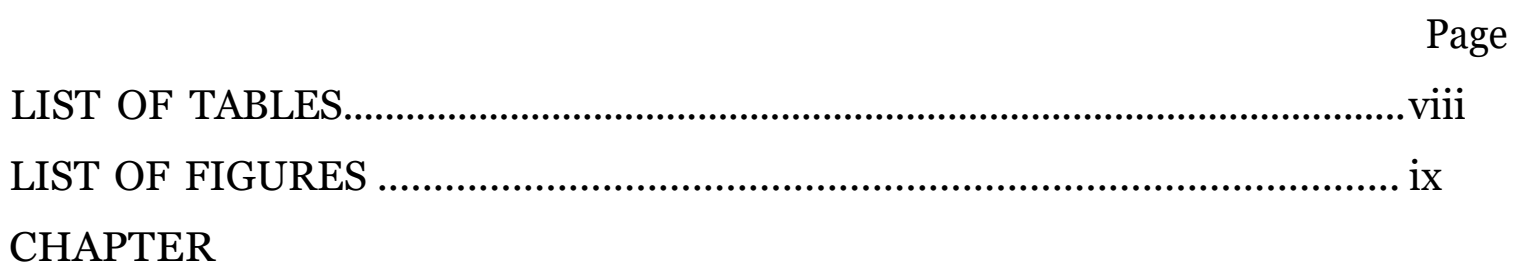

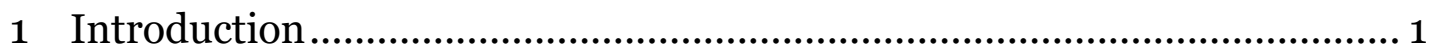

1.1 Cell Culture and Analysis ………………….................................. 1

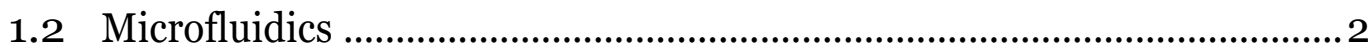

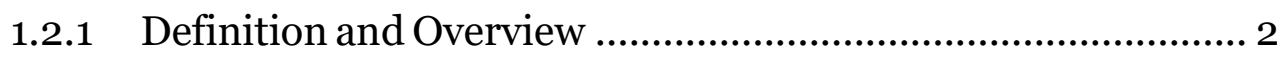

1.2.2 Advantages .............................................................................. 3

1.2.3 Disadvantages ...................................................................... 3

1.2.4 System Components.................................................................. 4

1.3 Impedance Spectroscopy ………………………………...........................

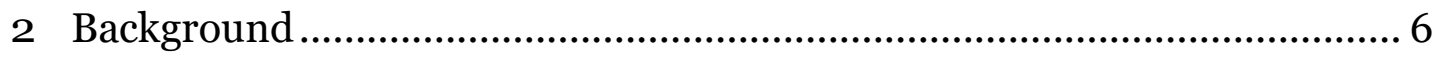

2.1 Fluid Properties ............................................................................ 6

2.1.1 Laminarity of Microflows ........................................................ 7

2.1.2 Hagen-Poiseuille Flow .................................................................

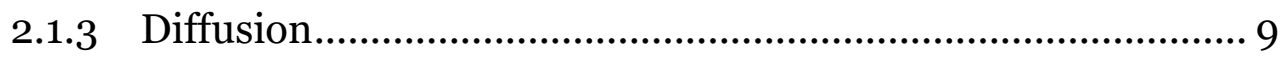

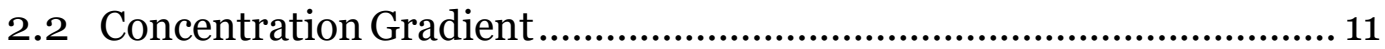

2.3 Electrical Impedance ....................................................................... 13

2.3.1 Parasitics ..................................................................................... 14

2.3.2 Electrical Properties of the Cell ................................................ 15

2.3.3 Electric Double Layer ................................................................. 16

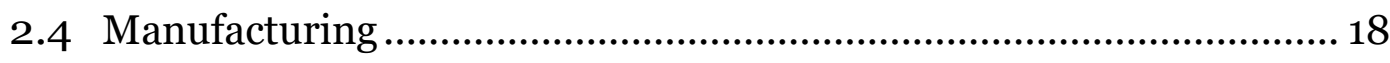

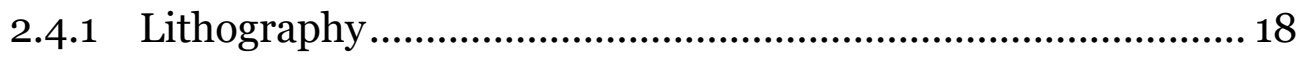

2.4.2 PDMS and Plasma Bonding ..................................................... 19

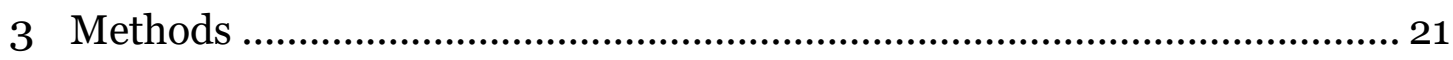

3.1 Microchip Design ............................................................................ 21

3.1.1 Design Considerations ............................................................. 23

3.2 Lithography: Creating the Mold.......................................................... 25

3.2.1 Characteristic Steps ..............................................................25 
3.2.2 The First Layer: Alignment Marks …………...........................29

3.2.3 The Second Layer: Shallow Channel Network ..........................30

3.2.4 The Third Layer: Deep Channel Network ................................30

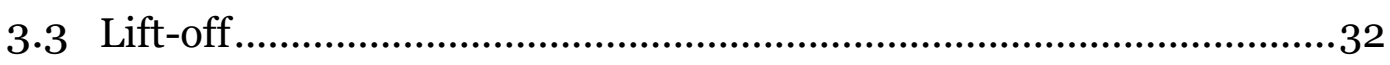

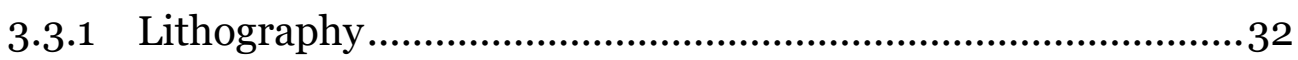

3.3.2 Metal Deposition ......................................................................34

3.4 PDMS and Plasma Bonding …......................................................... 37

3.4.1 Fluid Connection ...................................................................... 38

3.5 Safety Concerns .................................................................................39

3.6 Device Data Collection ....................................................................40

3.6.1 Instrumentation..................................................................40

3.6.2 Gradient Generator............................................................. 40

3.6.3 Ethanol Control ...................................................................... 41

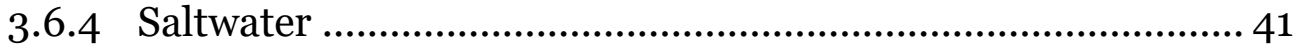

3.6.5 Cell Injection...................................................................... 41

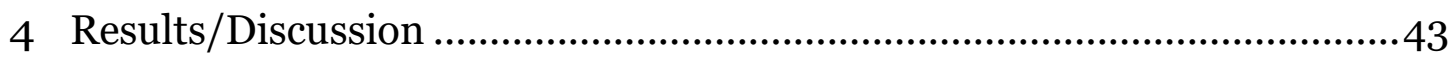

4.1 Manufacturing: SU-8 Mold …............................................................43

4.1.1 Alignment ...............................................................................43

4.1.2 Photoresist Spin.....................................................................43

4.2 Manufacturing: Electrode Array ........................................................44

4.2.1 Photoresist Spin......................................................................44

4.2.2 Sputtering............................................................................... 45

4.3 Characteristic Experiments...............................................................46

4.3.1 Concentration Generator...........................................................47

4.3.2 Impedance Measurements .....................................................49

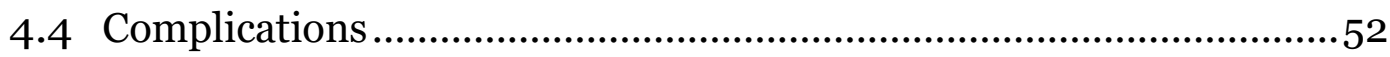

4.4.1 Device Leaks ......................................................................... 52

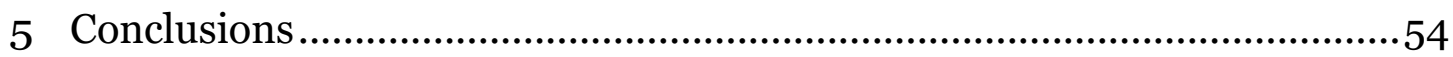

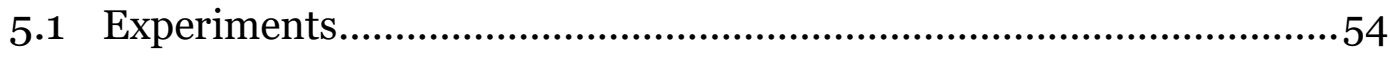

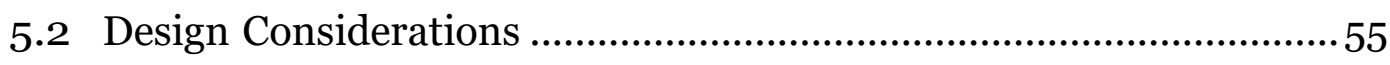

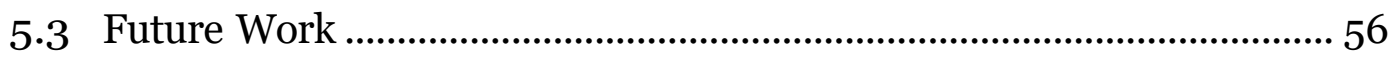

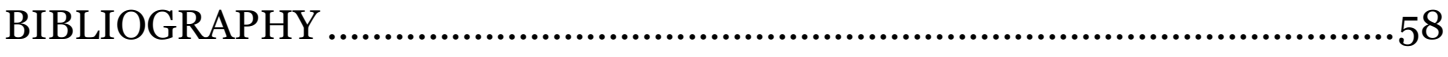




\section{LIST OF TABLES}

Table

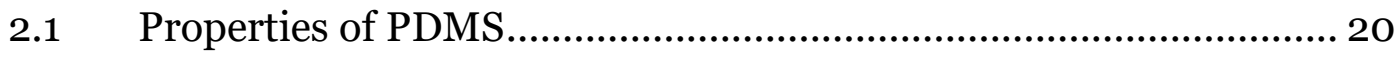




\section{LIST OF FIGURES}

Figure Page

1.1 Cell Presence in a Microchamber....................................................... 5

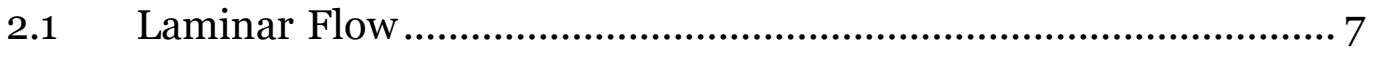

2.2 Christmas-Tree Gradient Generator ........................................... 12

2.3 Ideal Impedance Measurement Circuit ........................................... 14

$2.4 \quad$ Parasitic Electronics ...................................................................... 15

2.5 Lipid Bi-layer Membrane ………………...................................... 16

2.6 Electric Double-Layer .................................................................... 17

2.7 PDMS Chemical Structure............................................................20

3.1 Device Component Annotations........................................................ 21

3.2 Gradient Generator Design ...........................................................22

3.3 Cell Chamber and Cell Seeding Network Design.............................23

$3.4 \quad$ Fluid Delivery Network Design .......................................................24

3.5 Waste Collection Channel Design .....................................................24

3.6 Interdigitated Microelectrode Array Design .....................................25

3.7 Photoresist Spin Coater .................................................................26

3.8 CAD Drawing for Photomask ..........................................................2

3.9 Photoresist Exposure to Ultraviolet Light ........................................28

3.10 Alignment Marking Photomask ....................................................30

$3.1110 \mu \mathrm{m}$ Thick Microchannel Photomask ………................................. 31

3.12 $60 \mu \mathrm{m}$ Thick Microchannel Photomask.............................................32

3.13 Side-Wall Profile of Ma-N 1420 Photoresist.....................................33

3.14 Electrode Array Photomask...............................................................34

3.15 Machines for Physical Vapor Deposition .......................................... 35

3.16 Immersion in Photoresist Strip.......................................................... 37

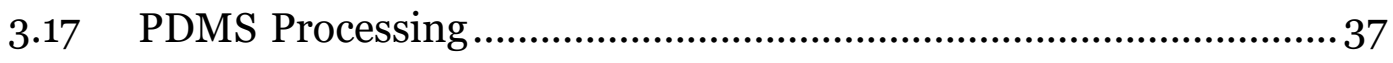

3.18 Tygon Tubing Connections ...............................................................39 


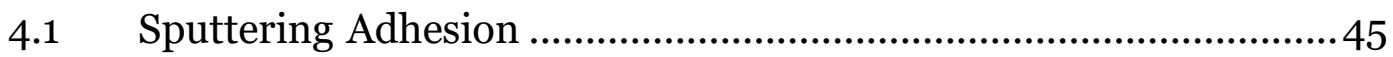

$4.2 \quad$ Sputtering Errors .........................................................................46

4.3 COMSOL Concentration Simulation...............................................4 47

$4.4 \quad$ Food Dye Experiment ...................................................................48

4.5 Fluorescein Concentration Experiment .............................................49

4.6 Ethanol Control Experiment ...........................................................49

4.7 Saltwater Impedance Comparison Experiment ................................50

$4.8 \quad$ Cell Injection Experiment.............................................................51 


\section{Chapter 1 INTRODUCTION}

\subsection{Cell Culture and Analysis}

When studying cellular behavior, whether in clinical diagnostics or academic research, cells are cultured, grown, or treated within a platform, such as a petri dish, culture flask, or bioreactor. Multiple methods have been developed for culturing, separating, and analyzing cell cultures [25], affecting metrics such as cell number, viability, and metabolite production for quantifying cell response to culture conditions [33]. Advances in bio-technology cell research in the past decades have been primarily in sterilization, materials, and the use of robotic automation to eliminate manual pipetting, increasing throughput and accuracy. The advancements, however have mainly enhanced test efficiency and accuracy and left much of the core of the processes the same.

These conventional methods come with several inherent limitations. Macroscale analysis is often labor-intensive and time consuming, and as the field of biotechnology grows and increases the demand for cell-based diagnostics, these traditional methods lack necessary throughput and make it difficult to quickly change and regulate cellular environments. Single-cell and dynamic analysis are difficult and are often done with conventional manual pipetting, decreasing accuracy and repeatability [25]. Furthermore, analysis of cellular components and the utilization of fluorescence-based dyes are often destructive to the sample, hindering subsequent experimentation [21]. To alleviate these limitations, we turn to the microscale, developing microfluidic platforms and "lab-on-chip" devices. Combining a variety of engineering disciplines with life science research, laboratory miniaturization hopes to reduce sample usage, cost, and testing inaccuracy while increasing cell throughput. 


\section{$1.2 \quad$ Microfluidics}

\subsubsection{Deftnition and Overview}

As the field of biotechnology continues to grow, larger numbers of experiments for DNA analysis, point-of-care diagnostics, and drug development are needed. Just as it transformed the world of electronics in the 1970's, moving systems down into the microscale has begun to transform the world of biotechnology as well [38]. Microfluidics is the field of study in which one manipulates fluids on the micron length scale. Initially, microfluidic devices began with analytical methods of gas-phase chromatography, high-pressure liquid chromatography, and capillary electrophoresis. As Cold War chemical threats and molecular biology genomics rose in the 1980's and 90's, microfluidic devices offered solutions for detection and DNA analysis spurring a rapid growth of academic research [37]. The first microfluidic devices were fabricated in silicon and glass in the 1990's using lithography techniques adapted from the microelectronics industry [38]. This incorporation of microelectromechanical systems (MEMS) manufacturing and biologics gave rise to the bioMEMS industry and subsequently evolved the field of microfludics as well. With bioMEMS, more complex fluid channel networks [28], valve flow controls [41], impedance detection [40], and even devices capable of full laboratory protocols are now possible.

In recent years, "organ-on-a-chip" based microfluidics have been developed to reproduce multiple physiological cell behaviorsin vitro. Models of the human lung [19], liver [22], and kidney [26] have been realised in vitro through the usage of bioMEMS and microfluidics. In many of these studies, device usefulness was characterized by easily measured and observed functionalities. Practically, however, in vitro models need to evaluate physiological responses to multiple biologic stimuli [18]. A microfluidic chiputilizing multiple culture wells could be one such approach tothis limitation, as it enables simultaneous cell studies under differing environmental conditions. 


\subsubsection{Advantages}

Utilizing nanoliters or less of fluid at a time, microfluidic platforms offer potentially higher throughput, lower cost per analysis, lower reagent and sample usage as well as improved portability, sensitivity, and reliability. Traditional macroscale cell sorting typically requires samples of $10^{5}-10^{6}$ cells due to losses during setup and operation, whereas microfluidic chips only need sample sizes in the $10^{2}$ range [16]. Microvolumes of sample allow for precise control of cell density, orientation, temperature, analyte concentration, and dosage. The physical dimensions of a microfluidic device allow for parallel experimentation on the same device and culture, enabling simultaneous cell assays and analyses [25].

Polydimethylsiloxane(PDMS) is the most common substrate for microfluidic devices. PDMS is optically transparent, non-conductive, elastic, and biocompatible. This polymer allows for ease in cell culturing, simultaneous, fluorescent imaging, and pneumatic valving.

Combining fluid mechanics, surface sciences, chemistry, biology, and often optics, electronics, and control systems areas of research, microfluidics has a great potential to impact a variety of industries such as pharmaceuticals, bio-defense, public health, point-of-care diagnostics, and agriculture.

\subsubsection{Disadvantages}

Constructing a device out of PDMS certainly has drawbacks. PDMS is hydrophobic in nature, leading to an adsorption of hydrophobic molecules (such as lipids) from culture media and resist aqueous fluid flow. PDMS is also porous, causing minor gas and water permeability. To alleviate these issues, PDMS is often surface treated [25] or designed around. 
While the field of microfluidics possess great potential to impact multiple interdisciplinary fields, microfluidic devices in general also come with several drawbacks. While microfluidic analyzers may have lower cost per analysis, the barrier-to-entry financially in this field is quite high. Commercial microfluidic device production currently requires clean room accessibility, often costing millions of dollars. Research, development, and resources for systems can be quite expensive compared to conventional counterparts. Furthermore, while the microfluidic chip itself might be space efficient, additional data acquisition hardware and power requirements (such as pumping mechanisms) can quickly turn a "lab-on-a-chip" into a "chip-on-a-lab”.

\subsubsection{System Components}

A modern microfluidic system is comprised of a variety of components: a method for moving or manipulating fluids, a series of delivery and channel networks, a chamber for analytes, and a modality for analytic techniques. These sub-systems can be internally integrated with the chip or externally sourced. The issue with internal integration is the increased design complexity of creating and interfacing these subsystems all with millimeter and sub-millimeter constraints. Power requirements, heat generation, cost, precision, and manufacturing difficulties need to be addressed for internal system components. The issue with external sourcing of sub-systems is an issue with size or portability. Using external pumps, sensors, and analytic software creates a microchip that needs an entire workbench to operate.

\subsection{Impedance Spectroscopy}

Impedance spectroscopy is a method of analysis that uses electricalimpedance measurements to characterize various system properties. Within microfluidic cell research, impedance spectroscopy can be used to quantify cell population change, 
fluid properties, or other changes in the cellular environment. For example, cell proliferation across electrodes should increase the overall system's complex impedance (equivalent circuit shown in Figure 1.1). Using these changes in impedance based on cell population, we can study how changes in cellular environments can influence cell growth. These impedance changes due to the presence of cells can be measured to quantify cells present in the system.

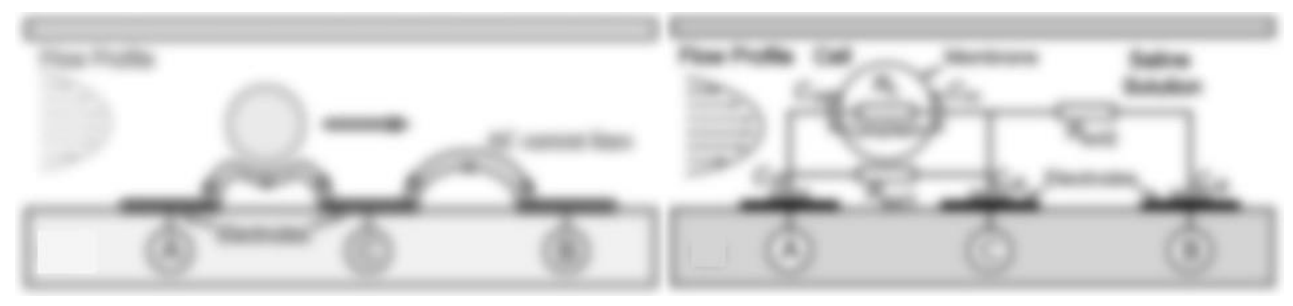

Figure 1.1 Cell Presence in a Microchamber: Image redacted from Figure 1 of Gawad et al. "Micromachined Impedance Spectroscopy Flow Cytometer for Cell Analysis and Particle Sizing" [13]. Please see source for full image

The principle of impedance spectroscopy was first demonstrated during fibroblast monitoring with an applied electric field in 1984 [14] and since then, impedance measurements of cell response and behavior have been made across interdigitated microelectrode arrays $[11,3]$. In 2004, Radke used impedance spectroscopy to detect Escherichia coli in samples $[29,30]$ and was able to make detections within 5 minutes. Recently, Rother et al. utilized impedance sensing to determine electromechanical connectivity between mammalian fibroblasts and cardiomyocytes [20].

While highly variable depending on electrode surface area, external circuitry, cell count, cell type, growth media, etc., one would expect higher overall impedance due to the cell's added capacitance on the system at-large. 


\section{Chapter 2 BACKGROUND}

We are interested in developing a microfluidic chip to measure cell impedance based on varying system environments. To that end, it is important to consider factors such as fluid properties within a microchannel, pressure-based flow, diffusive transport, the electric double layer, cell membrane impedance, and various microelectro-mechanical (MEMs) manufacturing techniques.

\subsection{Fluid Properties}

When developing a microfluidic system from the ground-up, it is important to consider the governing physics that influence design and system behavior. The following section will discuss various fluid properties that need to be considered in microfluid design.

Many characteristics governing microfluids are based on the macroscopic approach of continuum mechanics, that in every elementary volume of fluid there exist sufficient molecules to define fluid properties of interest, such as pressure, density, viscosity, specific heat, and temperature.

The Navier-Stokes Equation represents the conservation of momentum at any given fluid point [4]. In three-dimensional vector notation, the Navier-Stokes equation can be written as follows.

$$
\rho \frac{D \dot{v}}{D t}=-\dot{\nabla} P+\mu \dot{\nabla}^{2} \dot{v}+\dot{F}
$$

Where $\rho$ is density, $\dot{v}$ is fluid velocity, $P$ is pressure, $\mu$ is fluid viscosity, $\frac{D \dot{v}}{D t}$ is the time rate of change of a moving fluid, and $\dot{F}$ is the vector sum of applied forces.

Note that Equation 2.1 assumes that fluid is incompressible and Newtonian. Theseassumptions are viable for water at $20{ }^{\circ} \mathrm{C}$ under laminar flow [4]. 


\subsubsection{Laminarity of Microflows}

Fluid flow behavior is characterized by the ratio of viscous forces to inertial forces. When inertial forces dominate, flow becomes turbulent and random fluctuating vortices are allowed to develop. When viscous forces dominate, fluid flow lines become locally parallel and the fluid exhibits laminar flow. The laminarity of fluid flow is characterized by the dimensionless Reynold's number:

$$
R e=\frac{U D}{v}
$$

Where $U$ is average fluid velocity, $D$ is characteristic length, and $v$ is the fluid's kinematic viscosity.

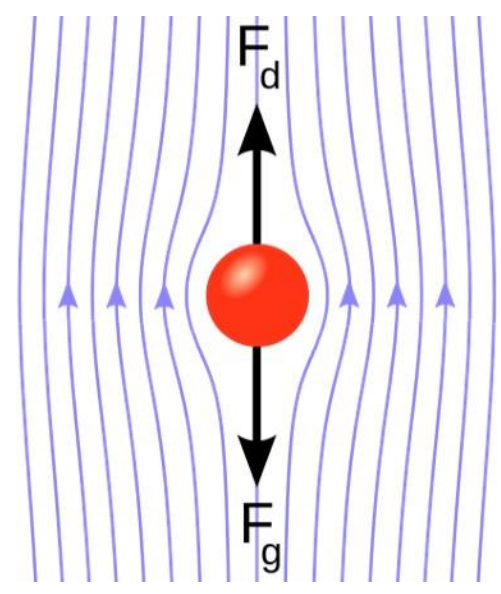

Figure 2.1 Laminar Flow: A sphere in Stoke's flow under very low Reynold numbers. [9]

Figure 2.1 demonstrates flow profiles with small Reynold's numbers $(<0.1)$. While turbulence is of consideration with macroscale fluid networks, microfluid channels often exhibit these laminar flow profiles, regardless of channel geometry. Owing to the characteristic length-scales and typical microfluidic flowrates, Reynold's numbers typically range between $10^{-4}$ and 1 [4]. 


\subsubsection{Hagen-Poiseuille Flow}

In cases of laminar flow profiles, there are analytical closed-form solutions to the Navier-Stokes Equation and an approximated solution for rectangular ducts. Given current manufacturing techniques for etching $\mathrm{Si}$, glass, or plastic in bioMEMS, microfluidic channels are often rectangular. When driven via pressure (i.e. syringe pumps), velocity profiles are parabolic in nature. For cylindrical ducts, pressure drop across a channel is given by,

$$
\Delta P=\frac{8 \mu U L}{R^{2}}
$$

Where $R$ in this equation represents a cylindrical radius, $U$ is averagefluid velocity, and $L$ is characteristic length.

Since current manufacturing techniques create rectangular microfluidic ducts, an approximation for the hydraulic radius is used, given by Equation

$$
R_{H}=\frac{2 S}{P}=\frac{2 a b}{2 a+2 b}=\frac{a b}{a+b}
$$

where $S$ is the channel cross section, $P$ is the perimeter, and $a$ and $b$ are the channel depth and width respectively.

The Hagen-Poiseuille equation is a physical law that explains thehydraulic behavior of pressure-driven flow through a circular channel in terms of driving pressure, flow rate, and hydraulic resistance. Analogously, Ohms Law $(V=I R)$ in electric circuit analysis describes the voltage drop and the electric current in a resistive conductor [27].

Combining these two laws, with pressure drop synonymous to voltage drop, volumetricflow ratetoelectric current, and hydraulic resistance $(R h)$ to electricresistance, we can write 


$$
\Delta P=Q R h
$$

While this analogy does not provide any information about velocity profiles or flow patterns, it does provide an excellent simplification to complicated microfluidic networks and necessary values such as maximum flow rates, pressure limits, and hydraulic resistances.

\subsubsection{Diffusion}

In simple fluids, a molecule will travel in a straight line until it collides with another molecule and changes direction. This distance until collision is known as the mean free path. Looking at a single molecule, it will continue to shift directions as it collides with other molecules. The path of this molecule over time is known as a particle's random walk or Brownian motion [4].

This concept of Brownian motion, first discovered byJ. Ingenhousz and R. Brown, forms the basic principle for diffusion. Each particle within a fluid exhibits this random walk effect. Over enough time, the average net displacement of each particle will have evenly dispersed throughout a given volume. This dispersion towards equilibrium is the phenomena of diffusion.

Fick's 1st Law relates a solute's mass flux to its concentration gradient

$$
\dot{J}=-D \dot{\nabla} c
$$

where Dis the solute's diffusion coefficient. Using Fick's 1st Lawand conservation of mass, we can write Fick's 2nd Law:

$$
\frac{\partial c}{\partial t}=D \Delta c+S
$$

where $\Delta \mathrm{c}$ is change in analyte concentration. 
The driving force behind diffusion is written as

$$
F_{\text {Diffusion }}=-\frac{1}{N_{A}}\left(\frac{\partial \mu}{\partial x}+\frac{\partial \mu}{\partial y}+\frac{\partial \mu}{\partial z}\right)
$$

where $N_{A}$ is Avogadro's number and $\mu$ is the chemical potential of the analyte:

$$
\mu=\mu_{\mathrm{o}}+\mathrm{RT} \ln (\gamma c)
$$

Where $\mathrm{R}$ is the gas constant, $\mathrm{T}$ is temperature in Kelvin, $\gamma$ is the specific weight of the fluid, and $c$ is the analyte's concentration.

We can then obtain

$$
F_{\text {Diffusion }}=-\frac{k_{\mathrm{B}} \mathrm{T}}{c}\left(\frac{\partial c}{\partial x}+\frac{\partial c}{\partial y}+\frac{\partial c}{\partial z}\right)
$$

which $F_{\text {Diffusion }}=F_{\text {Friction }}=C_{D v}$, where $C_{D}$ is the friction factor and $v$ is the stationary velocity. Thus

$$
v=-\frac{k_{\mathrm{B}} \mathrm{T}}{C_{D c}}\left(\frac{d c}{d x}+\frac{d c}{d y}+\frac{d c}{d z}\right)
$$

Comparing this to Ficks's Law, we obtain

$$
D=\frac{k_{\mathrm{B}} \mathrm{T}}{C_{D}}
$$

where $D$ represents a solute's diffusion coefficient, a key property in the design of diffusion-based fluid systems. For a sphere, we can use Stoke's drag, or $C_{D}=6 \pi \mu a$.

\section{Transport Phenomena}

In microfluidics and biotechnology, manipulation of target analytes such as DNA, proteins, cells, drugs, etc. is a key functionality of the device. Knowledge of trans- 
port phenomena within this space is necessary when designing a microfluidic device. Dependent of fluid velocity, microchannel dimensions, and the particles diffusion coeffecient (derived in section 2.1.3), transport of an analyte is dictated by diffusive and convective means, with the most dominant mechanism characterized bythe mass transport Peclet number, a dimensionless variable that gives the ratio of diffusive to convective flux.

$$
P e=\frac{U A}{D}
$$

where $A$ is the characteristic length of the microchannel, $U$ is the average fluid velocity, and $D$ is the diffusion coefficient from Equation 2.12.

A Peclet number less than 1 signifies that diffusion is the dominant mode of particle transport within the fluidic network. When this occurs, it is important to consider the time scale required for two fluids within a microchannel to reach steadystate equilibrium.

$$
t=\frac{A^{2}}{2 D}
$$

Using Equation 2.14, with a known fluid velocity, one can determine the characteristic mixing length $A$ needed for fluids to reach equilibrium. If equilibrium does not occur during mixing, incorrect concentrations will develop, negatively impacting results.

\subsection{Concentration Gradient}

A concentration gradient generator is capable of generating a wide range of concentrations. This network and concentration gradient is essential to the study of fluidic chemical properties and how they impact overall cell response. Microfluidics can be used to lower the time and space requirements to generate specific gradients, enabling simultaneous studies of multiple cellular environments and their respective responses to differing analyte concentrations [35]. 
Concentration gradients are necessary for a variety of biologic processes, such as development, immune response, and wound healing. While macroscale approaches could be used to generate these gradients even at the single-cell level, length scales in the microenvironment are on the order of microns, providing better gradient resolution and higher degrees of fluid control. With such small diffusive length, both channel lengths and time-to-dose are reduced as well.

Jeon et. al created a "Christmas tree" gradient generator and used it to deliver incrementally increasing concentrations of hydrofluoric (HF) acid to etch glass proportionally to the concentration of HF [10]. This gradient design has since been adopted in studies on chemical effects on cells [5],[31] and chemotaxis bacterial studies [12],[36]. An example of Jeon's Christmas tree generator is shown in Figure 2.2.

When designing a gradient generator, primary considerations are appropriate diffusive mixing lengths (based on fluid velocity and the diffusivity coefficient) and the total number of final mixing branches (for example in Figure 2.2, this generator will produce a gradient of 9 distinct concentrations). Using the aforementioned circuit analogy in subsection 2.1.2, concentration gradient channel dimensions can be easily designed without the use of complex simulations or additional experimentation.

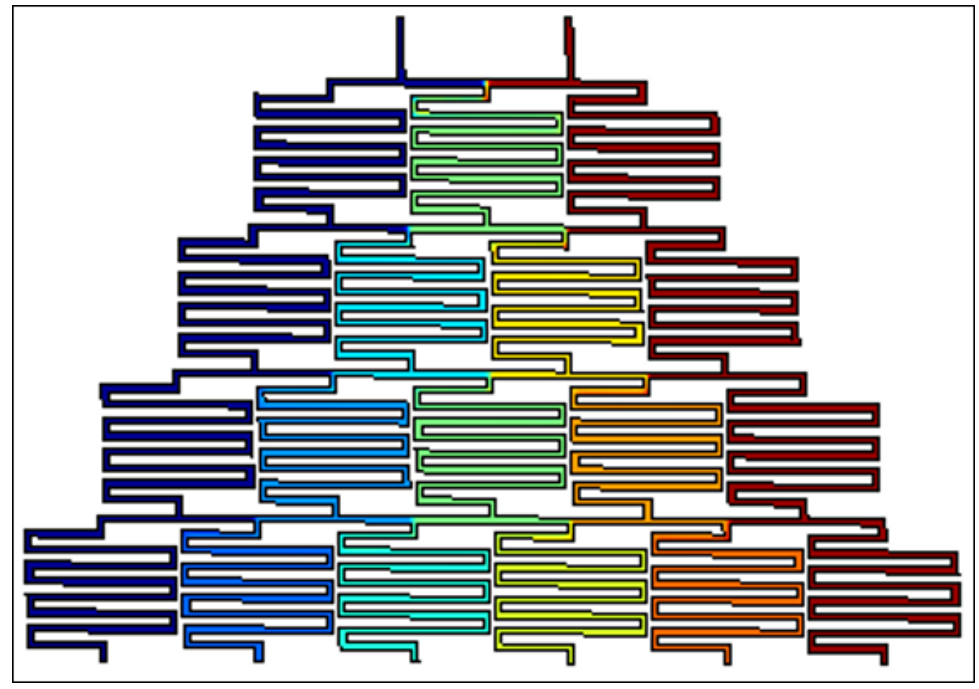

Figure 2.2 Christmas-Tree Gradient Generator: Red represents high analyte con- centration, whereas blue represents low concentration. 
Starting from the inlets, just like electrical current, flow rates are proportional to the summation of downstream resistance. Equalizing hydraulic resistance across all respective fluidic networks will cause an even flow distribution between each cell chamber.

\section{$2.3 \quad$ Electrical Impedance}

Impedance is a measurement of a circuit's resistance to current flow due to an applied voltage as a function of frequency. Using an alternating current (AC) voltage source, impedance becomes a complex value that combines resistive, capacitive, and inductive effects within a circuit, shown by Equation 2.15 .

$$
Z=R+j X
$$

where $Z$ is overall impedance, $R$ is real resistive effects, and $X$ is reactance, denoted by $j$, implying that this value is "imaginary".

Theamount of capacitance within a circuit is a frequency dependent valuedenoted by $Z_{C}={ }_{\rightarrow}^{1}$ Impedance due to capacitance is inversely proportional to frequency. When looking at the phase angle in AC circuit analysis, capacitance has a negative effect on phase angle. Inductance within a circuit is denoted by $Z_{L}=j \omega L$. Impedance due to inductance is directly proportional to rises in frequency. Inductance has a positive effect on phase angle, and when combined with capacitance, positive phase indicates stronger inductive effects, whereas negative phase indicates stronger capacitive effects. 
When measuring impedance, two oscilloscope probes are placed across the device under test (DUT). Current flows through the device, with relative voltage drop across the device being compared to voltage across an external resistor in the circuit. A frequency sweep is performed to measure the device's overall impedance across multiple frequencies. A basic impedance measurement setup can be shown in Figure 2.3.

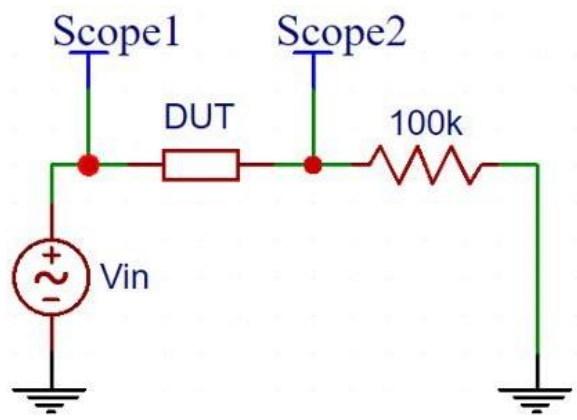

Figure 2.3 Ideal Impedance Measurement Circuit: Circuit for measuring system impedance under ideal conditions

\subsubsection{Parasitics}

No electronic system behaves in an ideal fashion. In every system, parasitic elements influence realistic measurements from their theoretical solutions. Node connections, such as clips, circuit boards, and breadboards can exhibit parasitic capacitance. Long or coiled wires from oscilloscopes and probes exhibit parasitic inductance. Even internal electronics from power supplies and computer systems add to a system's overall impedance. Depending on one's experimental setup, large parasitic elements in a system can significantly impact measurements by adding noise and reducing the system's overall sensitivity to variations in impedance. Adding parasitic elements into Figure 2.3, we see that a realistic circuit model looks closer to Figure 2.4. 


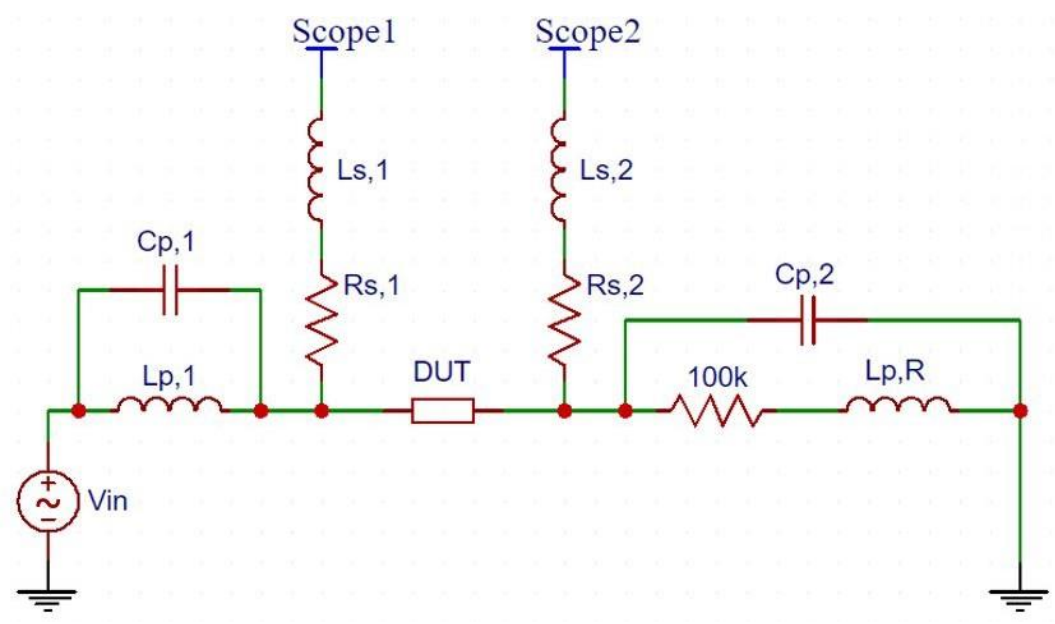

Figure 2.4 Parasitic Electronics: Impedance measurement circuit with added parasitic elements

\subsubsection{Electrical Properties of the Cell}

Mammalian cell membranes consist of a lipid bilayer containing double-chain phospholipid molecules, passive ion channels, and proteins throughout to allow transport of large molecules (such as glucose) into the cell's interior. Shown in Figure 2.5, in the 1940's, Hodgkin and Huxley carried out a series of electrophysiological experiments on a giant squid axon to gain a better understanding of the electrical properties of the cell membrane. They successfully characterized sodium and potassium channel potentials and membrane resistance and capacitance. This lipid membrane, $\sim 8 \mathrm{~nm}$ in thickness [17], is an extremely poor conductor and adds additional capacitive elements within a circuit. Furthermore, when cells adhere to a surface along focal regions they create cell-electrode gaps ranging from 0.15-0.5 $\mu \mathrm{m}[17]$. Combining the 

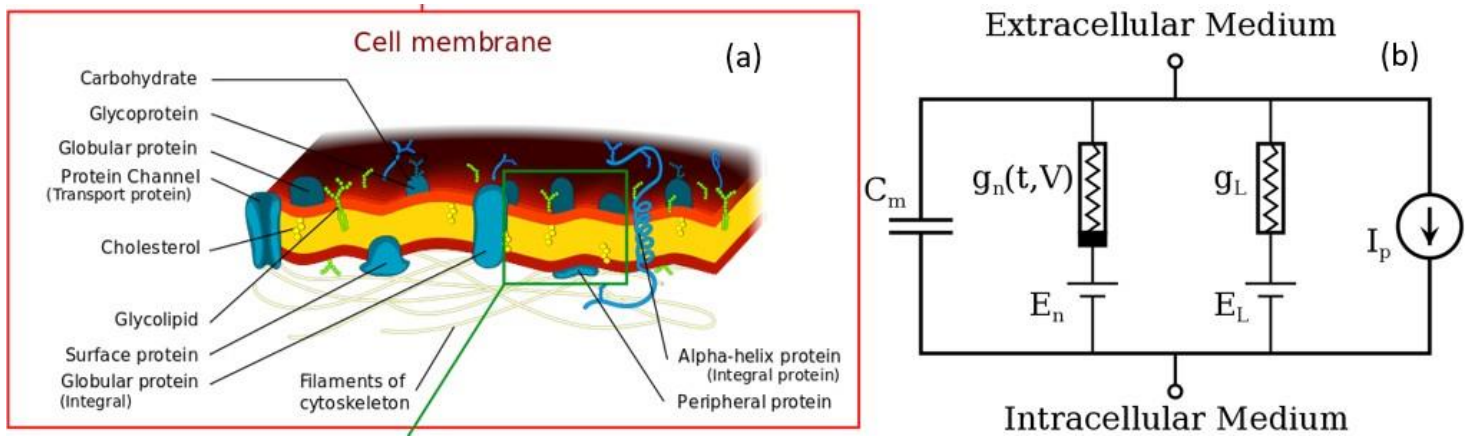

Figure 2.5 Lipid Bi-Layer Membrane: a) Representative picture of the various components of the cell mem- brane [6]. b) HogkinHuxley model of the electrical characteristics of the cell mem- brane [8]. $\mathrm{E}_{L}$ and $\mathrm{E}_{n}$ are the Nernst potentials for respective ion channels. $\mathrm{g}_{n}$, and $\mathrm{g}_{L}$ are the conductive elements for respective ion channels. $\mathrm{I}$ is a current traveling along the cell membrane, and $\mathrm{C}$ is the overall capacitance of the lipid bilayer.

capacitive membrane and adhesion gap with resistive cell elements (such as the nucleus in the cytoplasm), the presence of cells between electrodes should influence the overall impedance of the system.

\subsubsection{Electric Double Layer}

The electric double layer is an interface region formed whenever an electrode is immersed in an electrolytic solution. Functioning similar to a capacitive element, it is important to consider the electric double layer effects on the overall system impedance. Electrical properties and structure of this layer depend on a variety of factors, such as electrode material, surface oxides, surface area, solvent type, electrolyte type, and temperature [34].

In 1879, Helmholtz put forth the first model of the electric double layer [34]. This model assumed there existed a compact layer of ions in contact with the charged metal surface. Later, Gouy and Chapman suggested that this compact layer was in fact a diffuse layer of ions that extends some distance away from the metal surface, according to a Boltzmann distribution [34]. In 1924, Stern suggested that the interface 
contains both a rigid Helmholtz layer and a diffuse Gouy-Chapman one. In the 1950's and 60's, the role of the solvent was taken into account, and it was found that polar solvents, such as water, also interact with the charged metal surface [34].

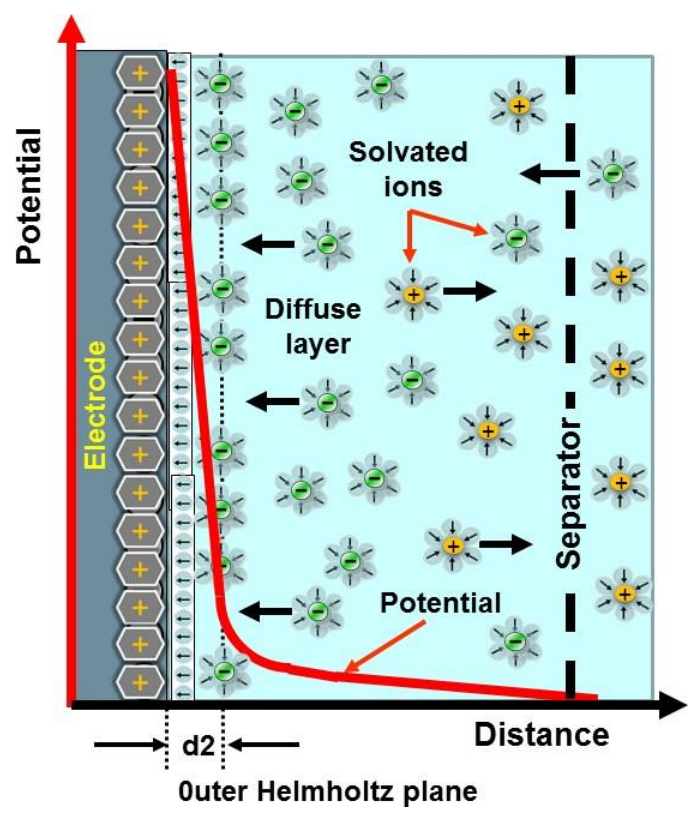

Figure 2.6 Electric Double-Layer: structure created by negatively charged metal surface [7]

Shown in Figure 2.6, the electric double layer consists of two planes. The first, the inner Helmholtz plane, consists of specifically adsorbed ions, located behind the layer of adsorbed water molecules. This inner plane represents the original rigid Helmholtz layer. The second, the outer Helmholtz plane, consists of hydrated ions in contact with the electrode surface. The diffuse layer develops beyond the outer plane, with ionic concentrations and potential decreasing exponentially with distance from the electrode [34].

While the impedance effects of the electric double layer in this project were not theoretically quantified, they are present in the system. Therefore, impedance measurements can be calibrated to a baseline measurement before and after biologic introduction. 


\subsection{Manufacturing}

\subsubsection{Lithography}

Lithography, invented in 1959, has been paramount for developing modern electronics and is the foundation for all integrated circuits. In the 1970's, lithography became commonplace in the semiconductor industry [39] and has since caused the field to expand exponentially, primarily in the field of smartphones and computer processors.

Derived directly from the microelectronics industry, manufacturing microfluidic platforms use this same process of lithography. It has enabled the smaller feature sizes and aspect ratios needed for fluidic networks. Photoresist, a photo sensitive polymer suspended in solvent, is spun onto a silicon wafer, typically 1-10 $\mu \mathrm{m}$ thick.

Exposure of the photoresist layer to ultraviolet light alters its solubility, allowing specific patterns to be transferred via a photomask [15]. The activated photoresist is then chemically removed using a developer solution, revealing the desired pattern transferred during UV exposure. Multiple layers of photoresist can be spun and exposed, then developed via a single development step [23].

SU-8 is a high contrast, negative-tone, epoxy-based photoresist that has gained much popularity for exploratory microfluidics. Upon exposure, SU-8 forms internal cross-links that prevent removal during development. This process creates a threedimensional microstructure of the photoresist on the surface of the wafer. In microfluidics, this structure serves several purposes. The first is usage as a negative mold to create necessary microchannel networks in a process called soft lithography. PDMS (polydimethyl siloxane) is mixed, poured, and cured over the microstructure mold. Channel networks are formed into the PDMS surface, which is then removedfrom the wafer, oxidized via plasma and bonded to another substrate. The second purpose 
involves a process known as lift-off. The microstructure serves as a protective layer during metallic sputtering [15]. Gas, typically argon or oxygen, is ionized and bombards a metallic target, releasing particles that then coat the photoresist and silicon wafer. The photoresist blocks metal from adhering to the silicon in specified areas. Upon chemical removal of the photoresist (lift-off), metal traces are left in areas of bare silicon during sputtering. These metal traces can form a series of interdigitated electrodes that can be placed alongside a fluidic network, creating a microscale device capable of measuring changes in impedance due to the system properties.

\subsubsection{PDMS and Plasma Bonding}

Early microfluidic platforms analyzing aqueous solutions were manufactured out of silicon and glass using conventional lithogrpahy adapted from the microelectronics industry[38]. Manufacturing these devices was expensive, time-consuming, and required highly specialized clean room environments, counter to the overall goals of microfluidics.

Polydimethylsiloxane, better known as PDMS, an organic silicone polymer, has gained large popularity in exploratory microfluidics research. PDMS has an intrinsicly hydrophobic surface consisting of repeating $-\mathrm{O}-\mathrm{Si}\left(\mathrm{CH}_{3}\right)_{2}-$ groups. While hydrophobic materials are generally a poor choice for aqueous solution analytics, exposure to oxygen plasma creates surface silanol $(\mathrm{Si}-\mathrm{OH})$ groups and destroys methyl $\left(\mathrm{Si}-\mathrm{CH}_{3}\right)$ groups, creating a temporary hydrophilic surface that can be properly wetted by aqueous solutions and polar solvents. These silanol groups can be further modified to create permanent hydrophilic surfaces, reduce nonspecific protein adhesion, or create cross-links for specific protein attachment [2]. A listing of relevant PDMS properties can be found in Table 2.1.

Irreversible seals can be formed between PDMS and PDMS, glass, silicon, polystyrene, 


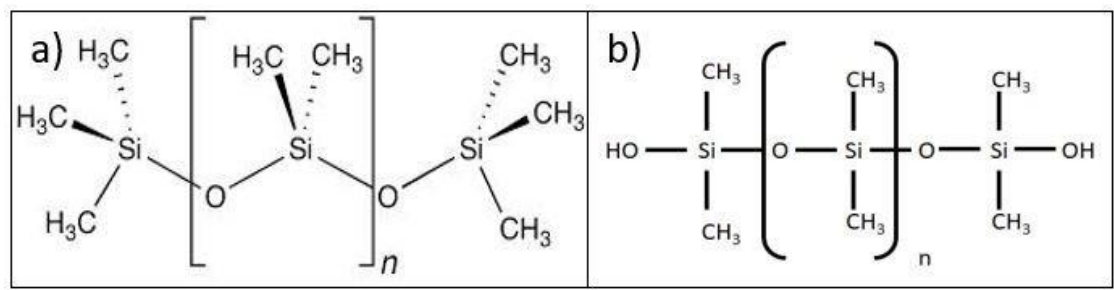

Figure 2.7 PDMS Chemical Structure: a) Chemical structure of PDMS. b) Chemical structure of PDMS after plasma oxidation [32]

polyethylene, or silicon nitride. When these materials are oxidized, polar-OH functional groups form covalent -O-Si-O- bonds with oxidized PDMS silanol groups, creating a bond stronger than the intrinsic bonds of PDMS itself. Keeping surfaces, dry, clean, smooth, and load-free during plasma bonding and a $70^{\circ} \mathrm{C}$ post-oxidation bake can greatly improve seal strength [24].

Table 2.1: Properties of PDMS [24]

\begin{tabular}{|l|l|l|}
\hline Property & Characteristic & Effect \\
\hline Optical & $\begin{array}{l}\text { Transparent w/ UV cutoff of } \\
240 \mathrm{~nm}\end{array}$ & $\begin{array}{l}\text { Optical usage between } 240 \text { and 1100 } \\
\text { nm }\end{array}$ \\
\hline Electrical & $\begin{array}{l}\text { Insulating, breakdown volt- } \\
\text { age of } 2 \mathrm{x}^{7} \mathrm{~V} / \mathrm{m}\end{array}$ & Allows for integrated circuitry \\
\hline Mechanical & $\begin{array}{l}\text { Elastomeric, Young's modu- } \\
\text { lus } \sim 750 \mathrm{kPa}\end{array}$ & $\begin{array}{l}\text { Surface conformation; reversible de- } \\
\text { formation allows for pneumatic actu- } \\
\text { ation }\end{array}$ \\
\hline Interfacial & $\begin{array}{l}\text { Lowsurfacefree energy, } \sim 20 \\
\text { erg/cm }{ }^{2}\end{array}$ & $\begin{array}{l}\text { Replicas easily removed from molds; } \\
\text { hydrophilic surface when oxidized } \\
\text { (SiOH functional group) }\end{array}$ \\
\hline Permeability & $\begin{array}{l}\text { Low permeability to liquid } \\
\text { water; permeable to gases } \\
\text { and nonpolar organic sol- } \\
\text { vents }\end{array}$ & $\begin{array}{l}\text { Channels maintain aqueous solu- } \\
\text { tions; gas transport allowed through } \\
\text { bulk; many organic solvents are in- } \\
\text { compatible }\end{array}$ \\
\hline Reactivity & $\begin{array}{l}\text { Inert; plasma exposure will } \\
\text { oxidize surface }\end{array}$ & $\begin{array}{l}\text { Unreactive with most reagents, sur- } \\
\text { face can be modified to be hy- } \\
\text { drophilic and reactive to silanes }\end{array}$ \\
\hline Toxicity & Nontoxic & $\begin{array}{l}\text { Can be implanted in vivo; allows for } \\
\text { mammalian cell growth }\end{array}$ \\
\hline
\end{tabular}




\section{Chapter 3 METHODS}

\subsection{Microchip Design}

Figure 3.1 represents a CAD outline of the two device footprints, the fluid channel networks and the microelectrode array pattern. The annotations in red can be used to identify various components of the device as discussed in the following sections.

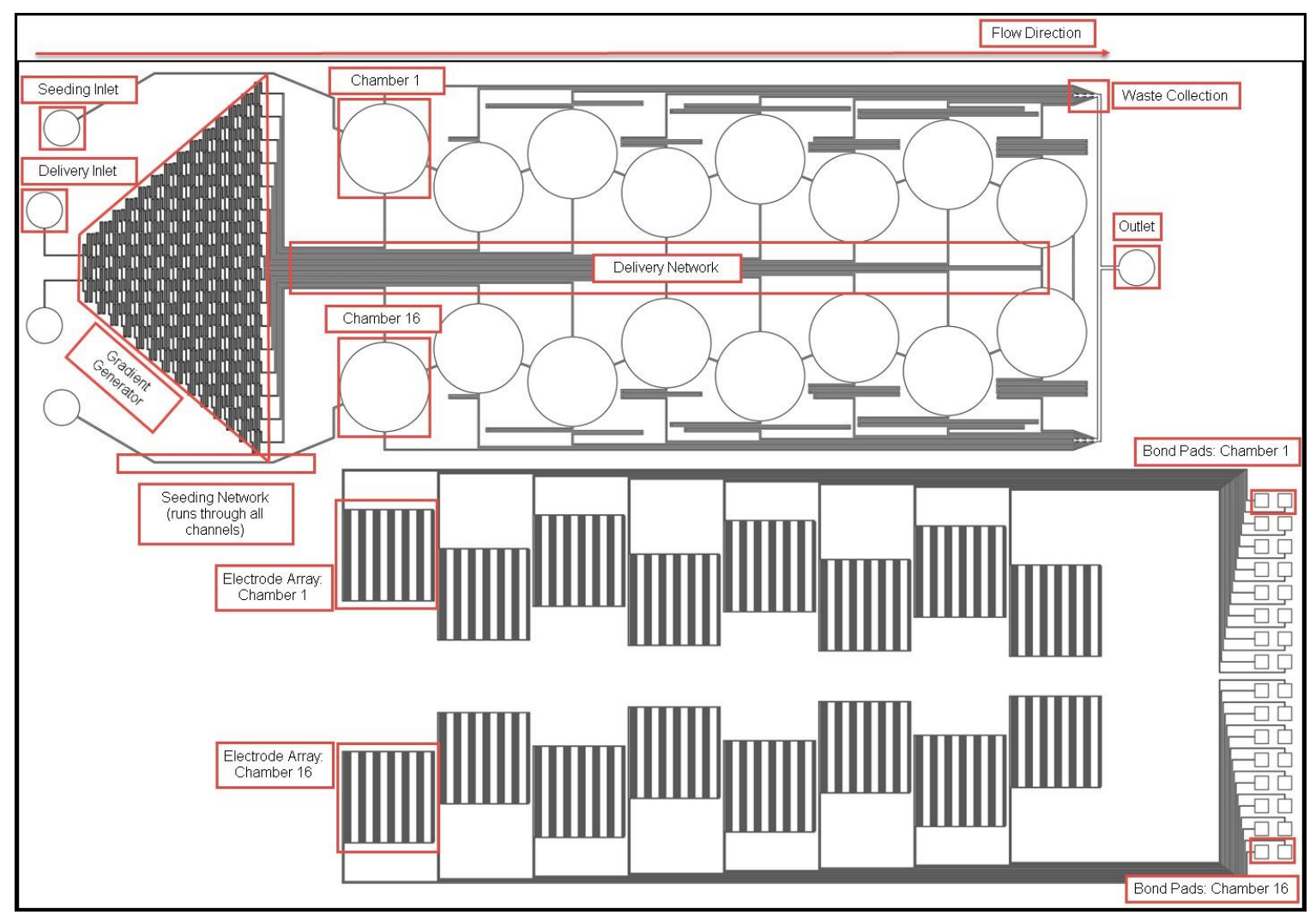

Figure 3.1 Device Component Annotations: Device footprint in CAD with annotations of various components in red. Top: Fluid channel network. Bottom: Microelectrode array pattern.

The microchip was designed to generate sixteen different solute concentrations across sixteen respective cell chambers. Within these chambers, a microelectrode array was patterned across each chamber base to measure the chamber's overall impedance, and, depending on differing solutes and/or biologics, measure changes in impedance. 
To generate a gradient of sixteen differing concentrations, Jeon's Christmas tree style was adopted. Gradient channels were designed to be $50 \mu \mathrm{m}$ in width, $10 \mu \mathrm{m}$ in depth, and each "mixer" to have a characteristic mixing length of $6 \mathrm{~mm}$ to ensure fluids have reached a diffusion equilibrium before the next mixing. Gradient generator design for this device is shown in Figure 3.2.
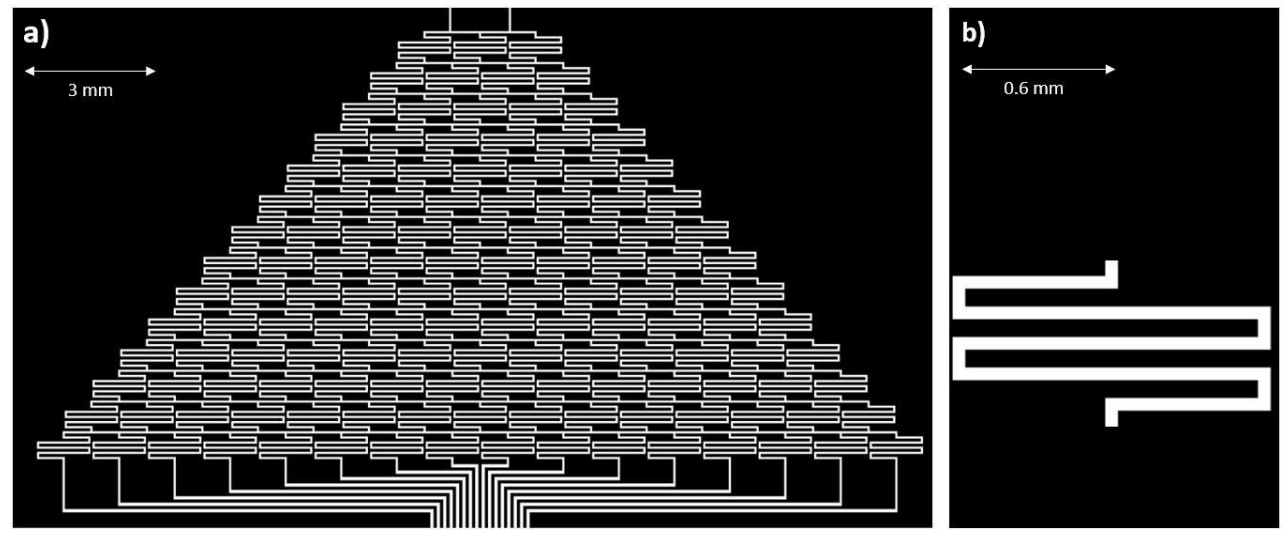

Figure 3.2 Gradient Generator Design: a) Full design for gradient generator b) Single characteristic mixer channel

Shown in Figure 3.3, cell chambers were designed to be $5 \mathrm{~mm}$ in diameter, 60 $\mu \mathrm{m}$ in depth, and contain an array of posts $75 \mu \mathrm{m}$ in diameter. These posts were designed to provide structural support due to the cell chamber's low aspect ratio (width height). A cell seeding network was placed between cell chambers (Figure 3.3) to allow cell injection prior to treatment. Channels were $75 \mu \mathrm{m}$ wide and $60 \mu \mathrm{m}$ in depth and were added to create a one-way path for cells throughout each of the chambers.

Fluidic networks, shown in Figure 3.4, into and out of the cell chamber were designed to be $75 \mu \mathrm{m}$ in width and $10 \mu \mathrm{m}$ in depth. The height difference between the fluidic network and the cell chamber is intended to prevent cell growth up the fluidic network by creating unfavorable cell growth conditions due to increased flow rate and wall shear in the delivery channels.

Cell waste collection, shown in Figure 3.5, begins at the end of the fluidic net- 
works and provides a universal collection channel, needing only one outlet port in the microchip. This channel is $200 \mu \mathrm{m}$ in width and $60 \mu \mathrm{m}$ in depth to minimize its

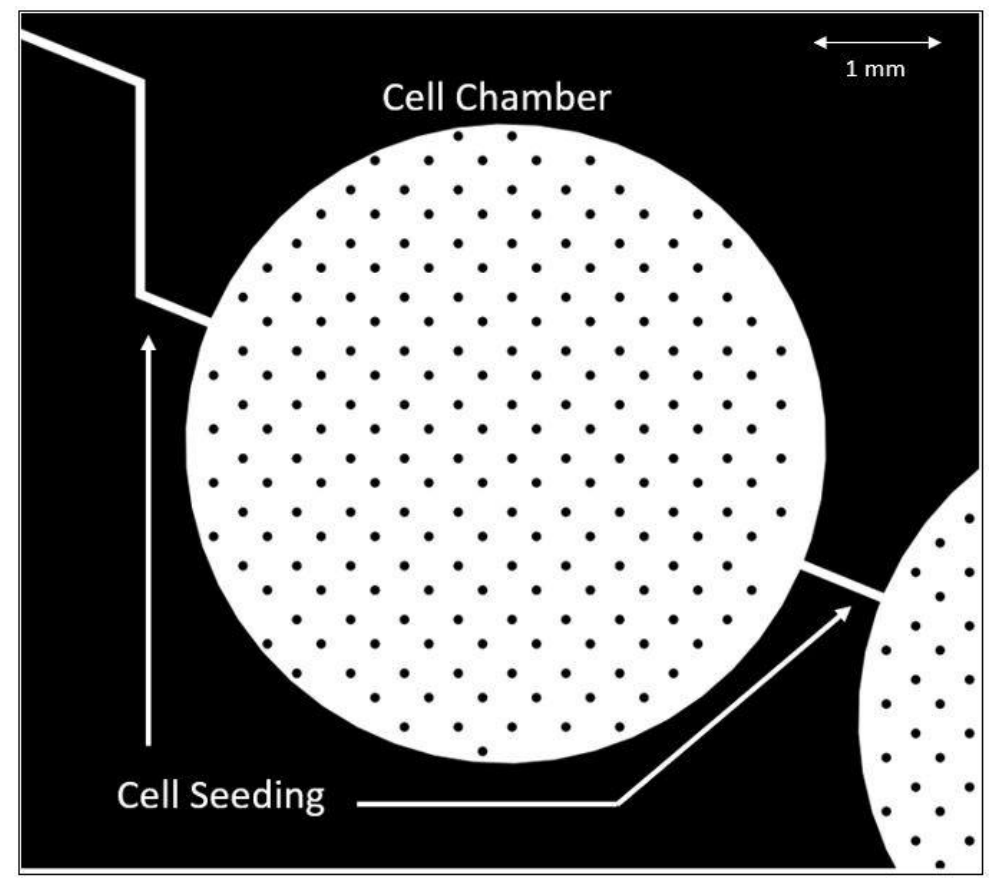

Figure 3.3 Cell Chamber and Cell Seeding Network Design:

Characteristic image of cell chamber and seeding network

impact on the overall fluidic resistance of the device.

The microelectrode array, shown in Figure 3.6, consists of an array of interdigitated electrodes $25 \mu \mathrm{m}$ in width and spaced $25 \mu \mathrm{m}$ apart. Larger gaps in the interdigitation were created to allow for potential microscopy quantification.

\subsubsection{Design Considerations}

Multiple factors such as material properties, manufacturing constraints, and experimental conditions must be taken into account during the design of themicrochip. Minimum feature size with SU-8 photolithography and available resources was 10 $\mu \mathrm{m}$. While constructing feature sizes at this length scale is possible, it increases the likelihood of error and reduces feature quality (eg., rounded edges or discontinuities). To avoid this, no geometries were designed under $10 \mu \mathrm{m}$. SU-8 material properties 
constrained maximum channel to depth to $100 \mu \mathrm{m}$.

Because the channels are shallow, a dimension width of less than $250 \mu \mathrm{m}$ was

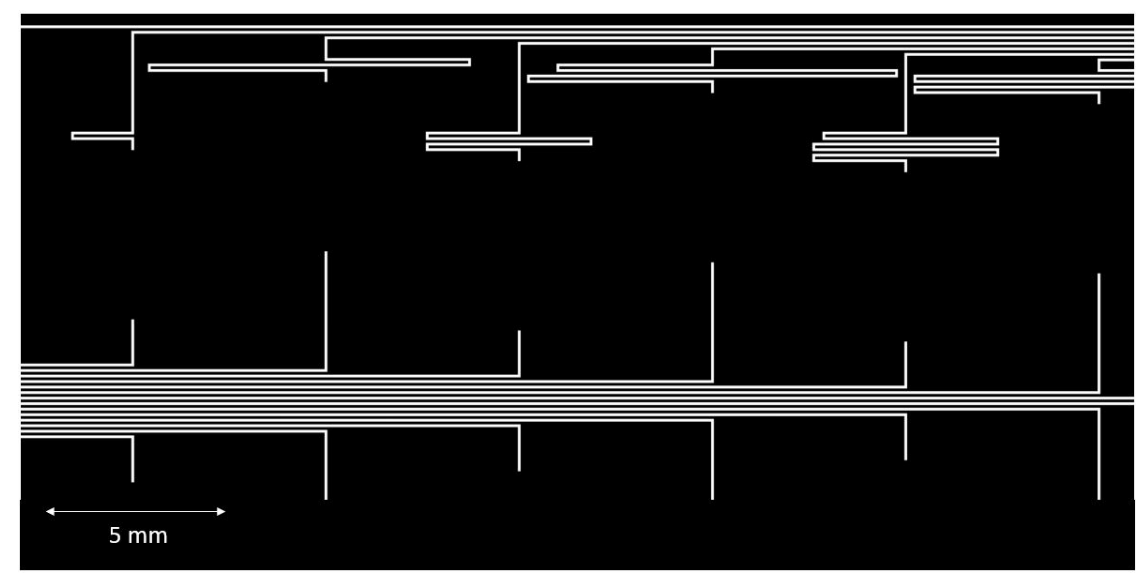

Figure 3.4 Fluid Delivery Network Design: Characteristic fluid path to cell chambers

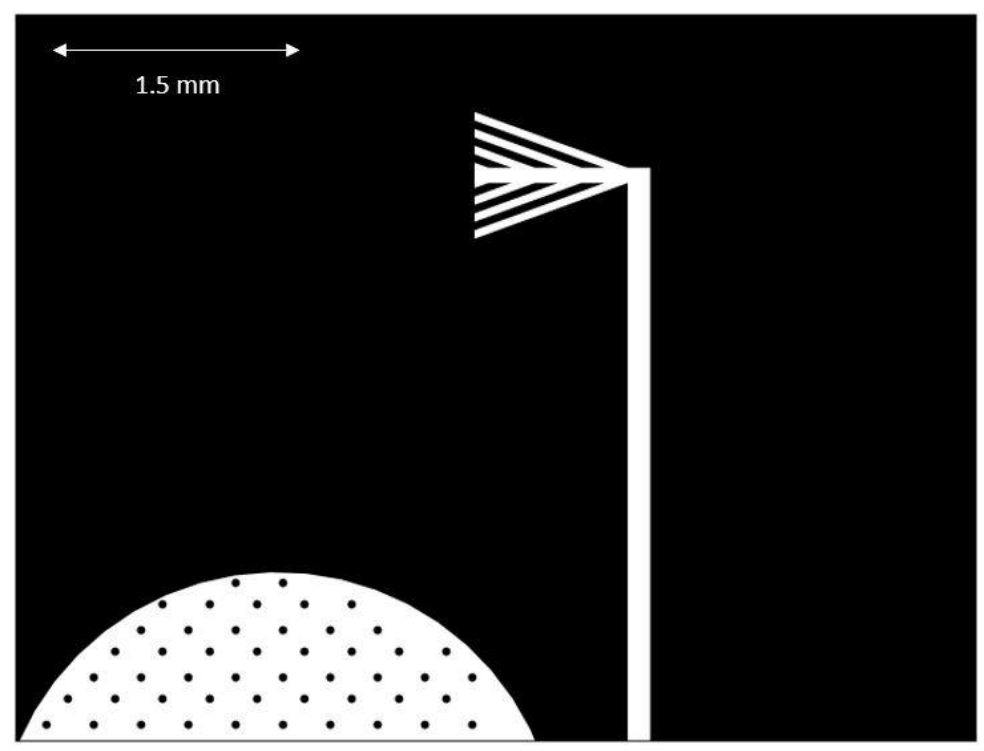

Figure 3.5 Waste Collection Channel Design: Characteristic cell waste collection network

observed to minimize "bowing" of the channel ceiling due to the weight of PDMS itself. Due to leaching and minor permeability of PDMS, no features were placed within $50 \mu \mathrm{m}$ of another to avoid any potential diffusion through PDMS. Furthermore, the maximum allowable footprint of the device was $25 \mathrm{~mm}$ by $75 \mathrm{~mm}$, as it was manufactured on a microscope slide to allow for potential microscopy. 

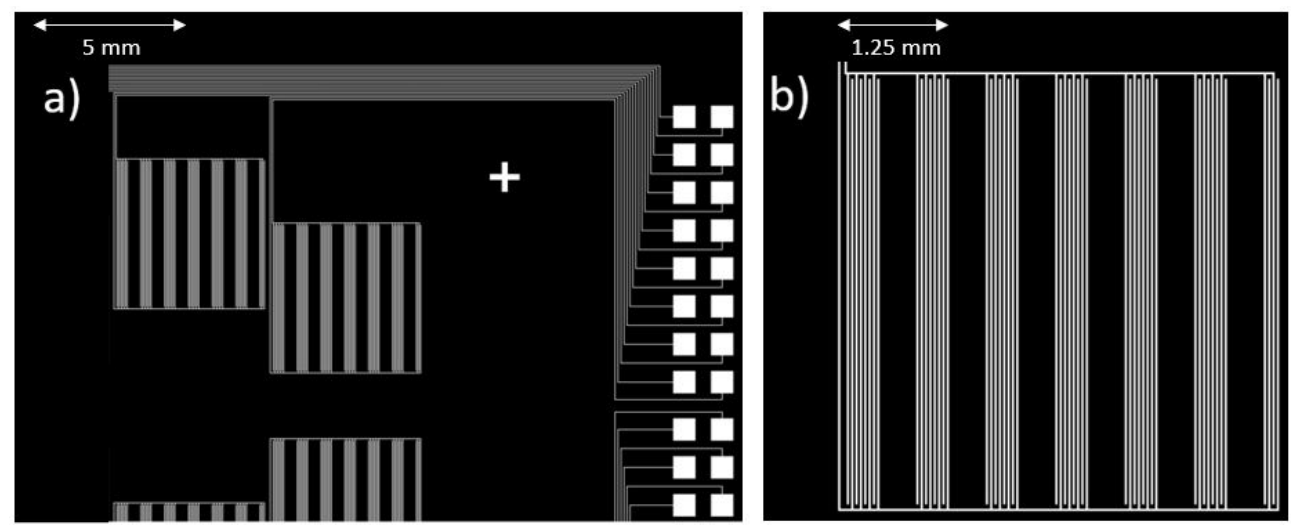

Figure 3.6 Interdigitated Microelectrode Array Design: a) Electrode arrays and bond pads b) Characteristic electrode array

\subsection{Lithography: Creating the Mold}

\subsubsection{Characteristic Steps}

The following section contains a general overview of the steps required in soft lithography. More details for each lithography process used can be found in later sections.

The first step in lithography is to clean the substrate. A silicon wafer was used as a flat, non-porous substrate for the photoresist mold. Wafers were first cleaned in piranha, a 9:1 mixture of $98 \mathrm{v} / \mathrm{v} \%$ sulfuric acid and $30 \mathrm{v} / \mathrm{v} \%$ hydrogen peroxide, at $70^{\circ} \mathrm{C}$ for 10 minutes. This is done to remove any organics potentially present on the wafer's surface. The wafers were then dipped in buffered oxide etch (BOE), a mixture of hydrofluoric (HF) acid and water, at room temperature for 1 minute. This is to remove any native oxide growth on the wafer's surface. Wafer's were placed in a spin-rinse-dry (SRD) tool to remove potential residual acids. A dehydration bake is then done at $150^{\circ} \mathrm{C}$ for 5 minutes on a hot plate to drive-off water from the surface. This is done as residual moisture will prevent photoresist adhesion to the silicon's surface.

The second step is to spin-on the photoresist. Using the Laurell spin coater shown 
in Figure 3.7, four milliliters of photoresist were poured over the wafer's surface and placed under vacuum. A slow spin speed was initially used to spread the resist evenly across the wafer surface. The spin speed was then increased to match the desired layer thickness according to the manufacturer's datasheet. The spin speed was then lowered to reduce stresses introduced to the photoresist during the high spin cycle. It is important to note that defects in the resist, such as poor adhesion or air bubbles, can be "reset" by immersing the wafer in acetone and rinsing with isopropyl alcohol and deionized water, then repeating the cleaning process from start. Post-spinning, the photoresist forms a thick layer on the edge due to its viscosity. This so-called edge-bead layer causes issues during exposure, and is removed by using a razorblade to and wipe the outermost surface edge.

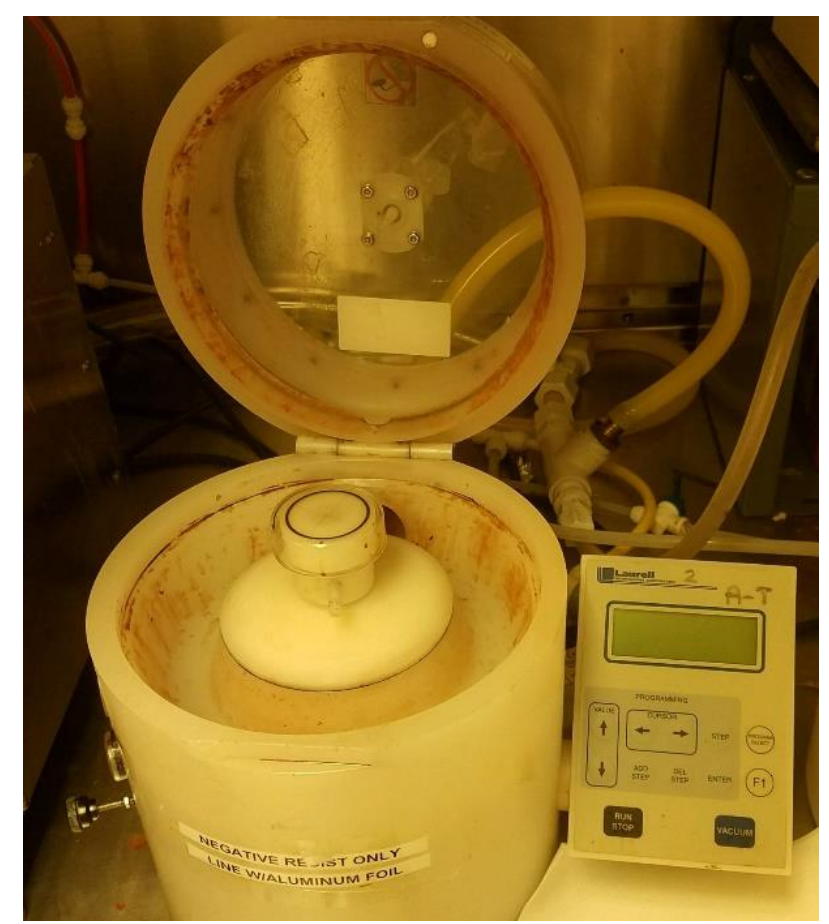

Figure 3.7 Photoresist Spin Coater: Spin coat machine used to spin-on various layers of photoresist

The third step is to soft-bake the wafer. This is done to evaporate solvent and promote thermal stability. Wafers were placed on a hot plate at $65^{\circ} \mathrm{C}$ and increased to the desired temperature for the resist $\left(95-100^{\circ} \mathrm{C}\right)$ for approximately 2 minutes. The 
temperature was then passively cooled down to $65^{\circ} \mathrm{C}$ and then to room temperature. This temperature ramp cycle is done to minimize thermal stresses on the photoresist layer.

The fourth step is ultraviolet (UV) exposure. A two-dimensional CAD drawing (Figure 3.8) of the microchip was sent to CAD/Art Services, where it was then separated into separate layers and printed on mylar sheets with 20,000 dpi resolution. This mylar photomask serves as a protective layer against UV exposure to the photoresist. Three layers were used for the SU-8 master mold: alignment marks, the 10 $\mu \mathrm{m}$ thick microchannels, and the $60 \mu \mathrm{m}$ thick microchannels. A fourth photomask was used in a later process to pattern the electrode array as well.

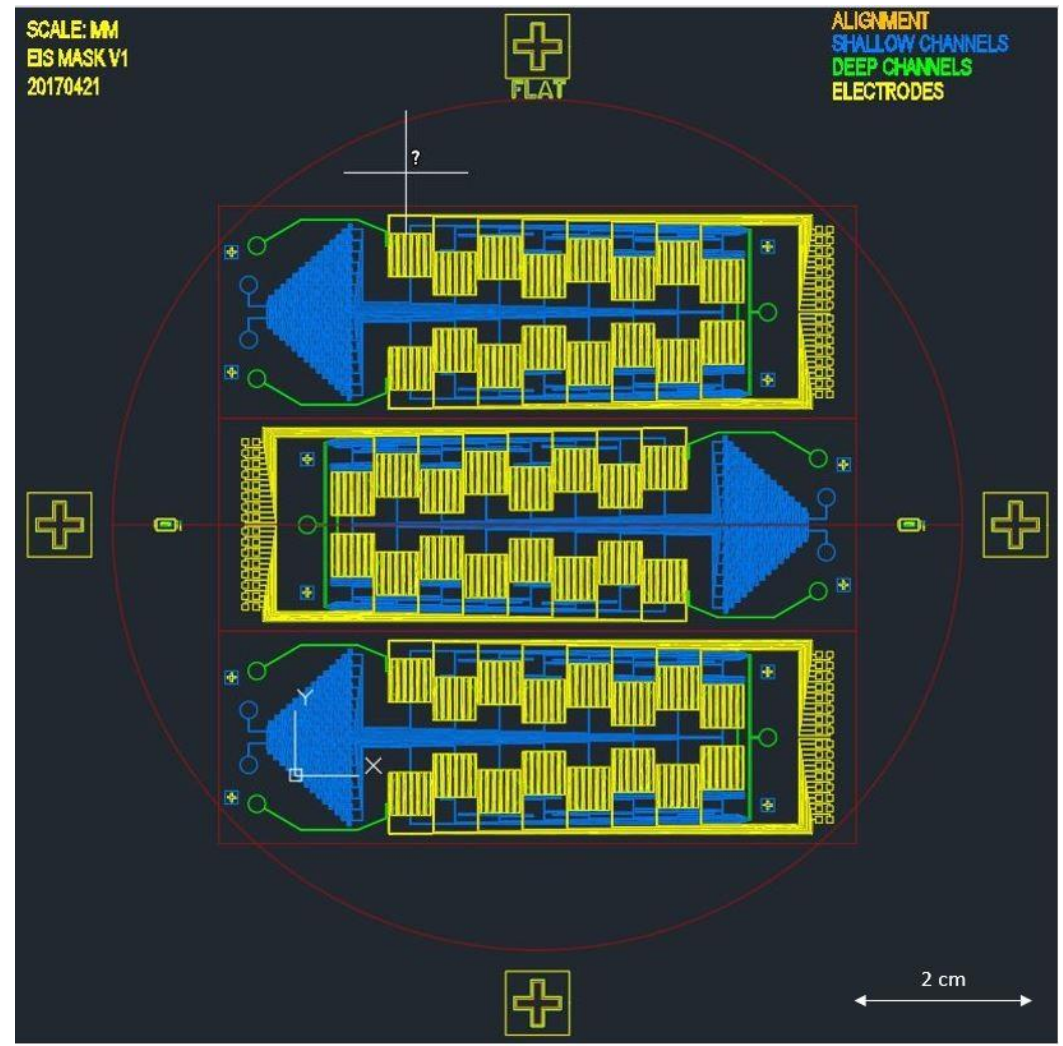

Figure 3.8 CAD Drawing for Photomask: CAD File used for mylar photomask production 


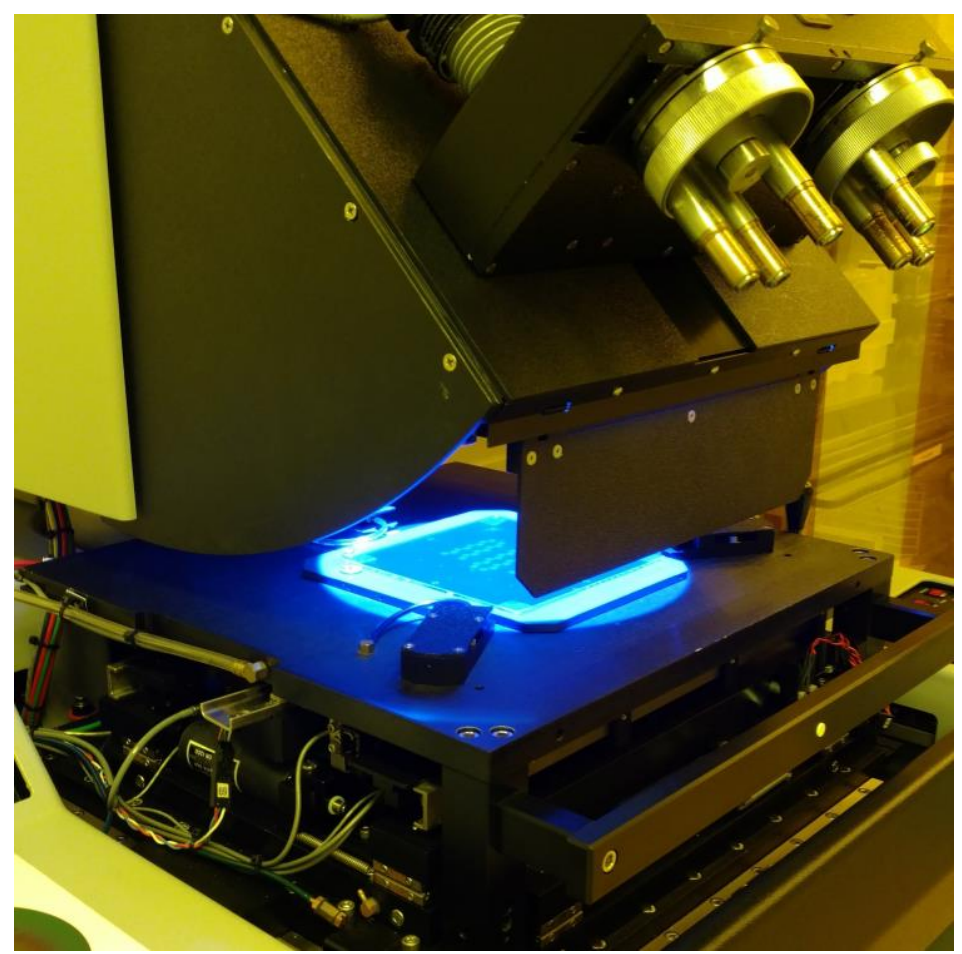

Figure 3.9 Photoresist Exposure to Ultraviolet Light: Wafer during UV exposure

Figure 3.9 shows a wafer covered by a mylar photomask undergoing UV exposure. Wafers were placed $10 \mu$ m away from the respective mylar photomask and glass plate. Exposure dosage was set to $15 \mathrm{~mW} / \mathrm{cm}^{2}$ with an h-line mercury arc lamp. Exposure times varied based on the resist itself and its layer thickness. Most of the photoresists used in this process are negative-tone resists, meaning that UV exposureforms crosslinks within the resist, rendering it insoluble to the developer solution. This step forms the desired pattern from the photomask onto the photoresist.

The fifth step is a post-exposure bake (PEB). A similar process to the post-spin soft-bake, a PEB is done to reinforce the cross-links created during UV exposure. Patterns should become visible during this step. While some resists do not require this step, failure to do so when needed will remove all photoresist from the surface, not just the areas of interest.

The final step is development. Developer solutions used were the those premade by the photoresist manufacturer. In the case of negative-tone resists, 
unexposed areas are removed during this process. Development times are highly variable based on resist type, layer thickness, and developer age, with manufacturer datasheets used for estimates. Wafers were immersed and slightly agitated within the developer for the allotted time. Upon removal, they were rinsed with isopropyl alcohol and/or deionized water. If a milky-white resist was present on the surface during the rinse, the wafers were immersed in developer in 30 second increments until a clean rinse was achieved. The wafers were then spin-rinsed-dried and set forstorage.

\subsubsection{The First Layer: Alignment Marks}

Before photoresist spin-on, the wafers were placed in an oxidation furnace at $1100^{\circ} \mathrm{C}$ to grow a thin uniform layer of oxide over the wafer's surface. The first layer of the photoresist aids in creation of the alignment structure. For this, AZ1529 was used instead of SU-8, as SU-8 is not easily removed. AZ-1529, unlike SU-8, is a positive-tone resist, meaning that UV exposure renders it soluble to developer solution. Four milliliters of resist was poured over the wafer and spun-on at $400 \mathrm{rpm}$ for 15 seconds to spread the resist evenly, then increased to $3000 \mathrm{rpm}$ for 30 seconds and a $300 \mathrm{rpm} 10$ second spin. The resist was then exposed to the first photomask: the alignment layer, as depicted in Figure 3.10. After being developed in CD-26, a developer by MicroChem, resist was removed in specified areas.

This process left photoresist over the surface oxide at the alignment marks. The wafer was then dipped in BOE for 5 minutes to remove the oxide, with the photoresist serving as a protective layer during removal, leaving oxide structures to serve as the alignment layer. An oxide was chosen for this layer as it provided the greatest optical contrast in future alignment steps. The wafer was then spin-rinsed-dried and dehydration baked in preparation for the next photoresist layer. 


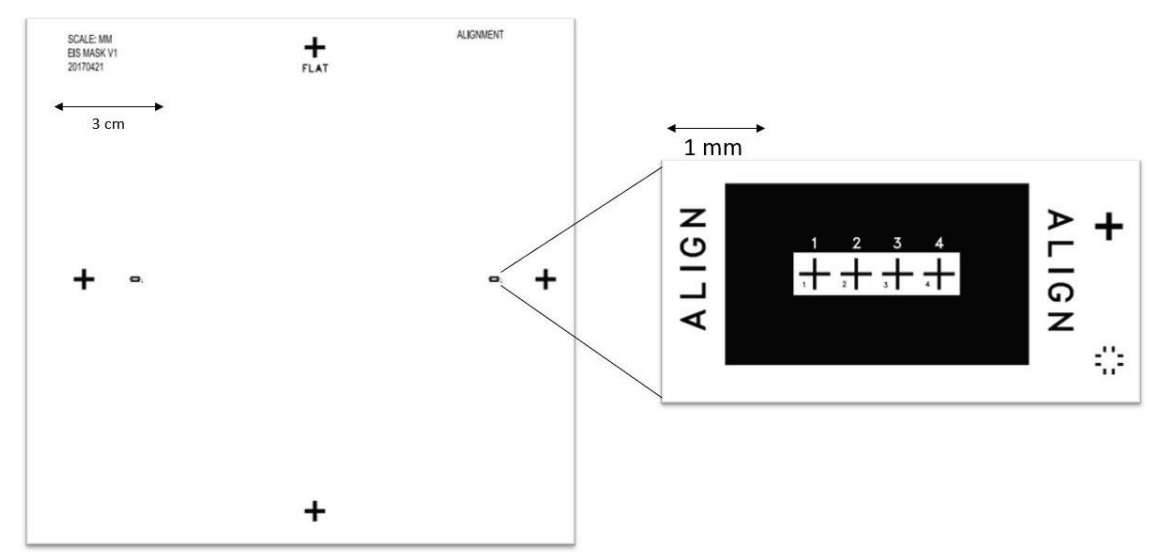

Figure 3.10 Alignment Marking Photomask: Mylar photomask used for the first layer of AZ-1529 photoresist. Pat- terns the alignment marks used for future resist layers.

\subsubsection{The Second Layer: Shallow Channel Network}

The second layer of photoresist serves as the $10 \mu \mathrm{m}$ thick mold for microchannels. The layer will form the concentration gradient and fluid networks to and from the cell chambers. SU-8 2007, a negative-tone photoresist by MicroChem, was used for this layer. The resist was removed from refrigeration and brought to room temperature, then was spun-on to the surface at 200 rpm for 25 seconds, 500 rpm for 10 seconds, $1500 \mathrm{rpm}$ for 30 seconds, and $300 \mathrm{rpm}$ for 10 seconds for a target $10 \mu \mathrm{m}$ thick layer. After edge bead removal, the wafer was soft-baked at $95^{\circ} \mathrm{C}$ for 2 minutes and 30 seconds, with a temperature ramp starting from $65^{\circ} \mathrm{C}$ to reduce thermal stresses. The wafer was exposed to the second photomask layer (Figure 3.11) for 8.3 seconds using the oxide alignment marks for guidance. This layer does not get developed until after the next layer of photoresist.

\subsubsection{The Third Layer: Deep Channel Network}

The third layer of photoresist serves as the $60 \mu \mathrm{m}$ thick mold for microchannels. This layer will form the cell seeding network, the cell chambers, and the waste collection network. SU-8 2050 was used for this layer, as its higher viscosity compared to 


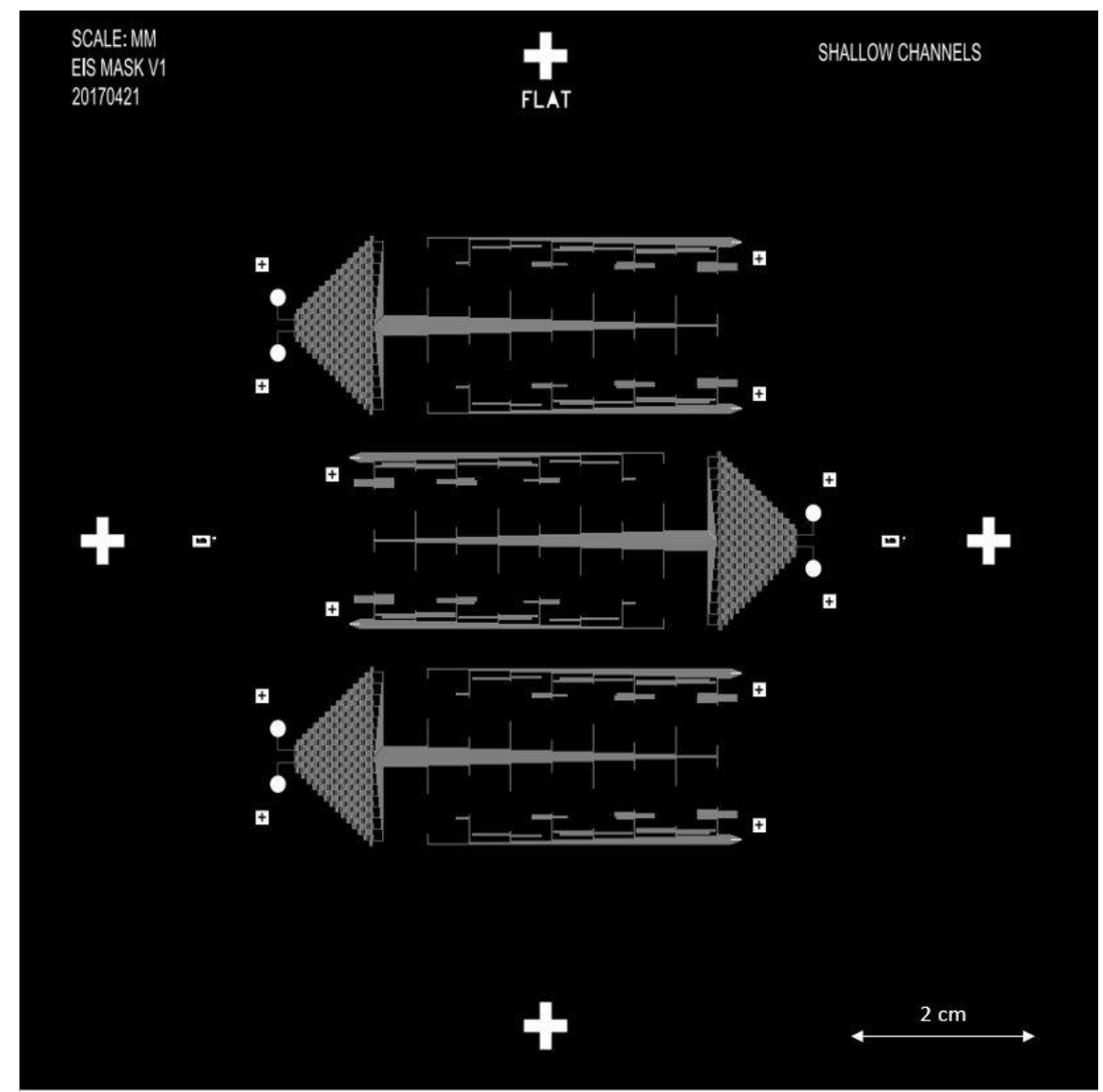

Figure 3.11 $10 \mu \mathrm{m}$ Thick Microchannel Photomask: Mylar photomask for the SU-8 2007 layer. Patterns the gradient generator and fluid networks

SU-8 2007 allows for thicker channel geometries. The wafer was soft-baked at $95^{\circ} \mathrm{C}$ for 6 minutes, using appropriate $65^{\circ} \mathrm{C}$ temperature ramps as well. After being exposed to the third photomask layer (Figure 3.12) for 11.4 seconds, the wafer was developed in MicroChem's SU-8 developer for 6 minutes, removing unexposed SU-8 2007 and SU-8 2050. Isopropyl alcohol was used to clean the developer solution, where the wafer was then rinsed with deionized water, and spin-rinse-dried. 


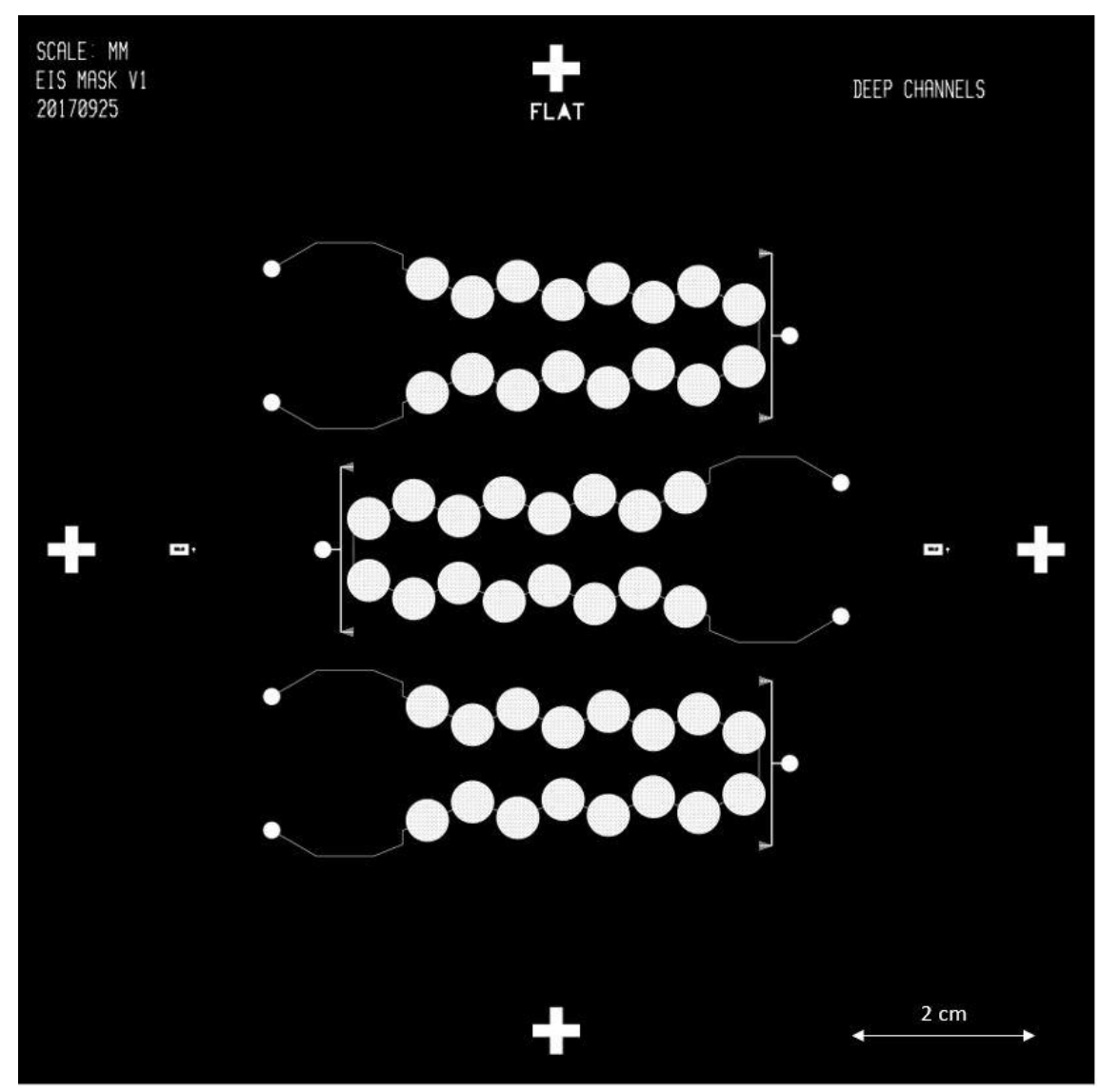

Figure 3.12 $60 \mu \mathrm{m}$ Thick Microchannel Photomask: Mylar photomask for the SU-8 2050 layer. Patterns the cell seeding network, cell chambers, and waste collection

\subsection{Lift-off}

\subsubsection{Lithography}

A glass wafer was used as the substrate for the lift-off process. Due to its transparency, patterning the microelectrode array over glass allows for the ability to take microscopy measurements if needed. The wafer was first cleaned in piranha at $70^{\circ} \mathrm{C}$ for 10 minutes and buffered oxide etch (BOE) for 30 seconds. After a spin, rinse, and dry, the wafer underwent a dehydration bake at $200^{\circ} \mathrm{C}$ for 10 minutes.

The photoresist ma-N 1420 by MicroChem was used for this process. This resist was chosen over SU-8 due to its side wall profile. Upon development, SU-8 forms 
straight $90^{\circ}$ side walls, ma-N 1420 forms an "undercut" along its side wall, as shown in Figure3.13. This undercut profileenablesalaterstep of resistremovaland subsequent metal adhesion to the glass wafer.

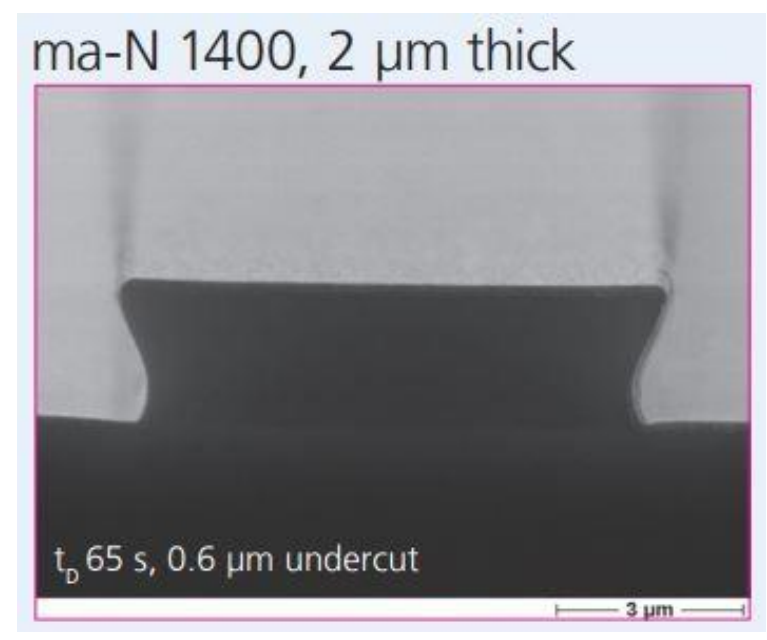

\section{Figure 3.13 Side-Wall Profile of Ma-N 1420 Photoresist:} Sample Ma-N 1420 photoresist side wall profile [1]

Like AZ-1529, ma-N 1420 also has poor adhesion to Si-based substrates. HMDS $80 / 20$ primer first was spun-on to the surface at 3000 rpm for 30 seconds, then maN 1420 was spun-on using the same spin-cycle for HMDS, but with 2000 rpm as the characteristic spin speed, creating a $2.5 \mu \mathrm{m}$ thick layer of resist. Ma-N 1420's viscosity is much lower than SU-8, and edge bead removal is not required. Post-spin, the wafer was soft-baked at $100^{\circ} \mathrm{C}$ for 4 minutes. The increased temperature and time of the bake helps increase the resist's overall thermal stability. The wafer was exposed under the photomask in Figure 3.14 for 30 seconds, with a black backing on the wafer to prevent reflected light from exposing the resist's underside (recommended for transparent substrates). Development was done using ma-D 533/S by MicroChem for 90 seconds.

Post-development the wafer underwent a flood exposure process. The wafer was exposed to a blank mask for 30 seconds 3 times, with 2 minutes of rest in between each dosage. This process is done to aid in the undercut profile formation of the 
photoresist and to thermally stabilize the layer during subsequent processing.

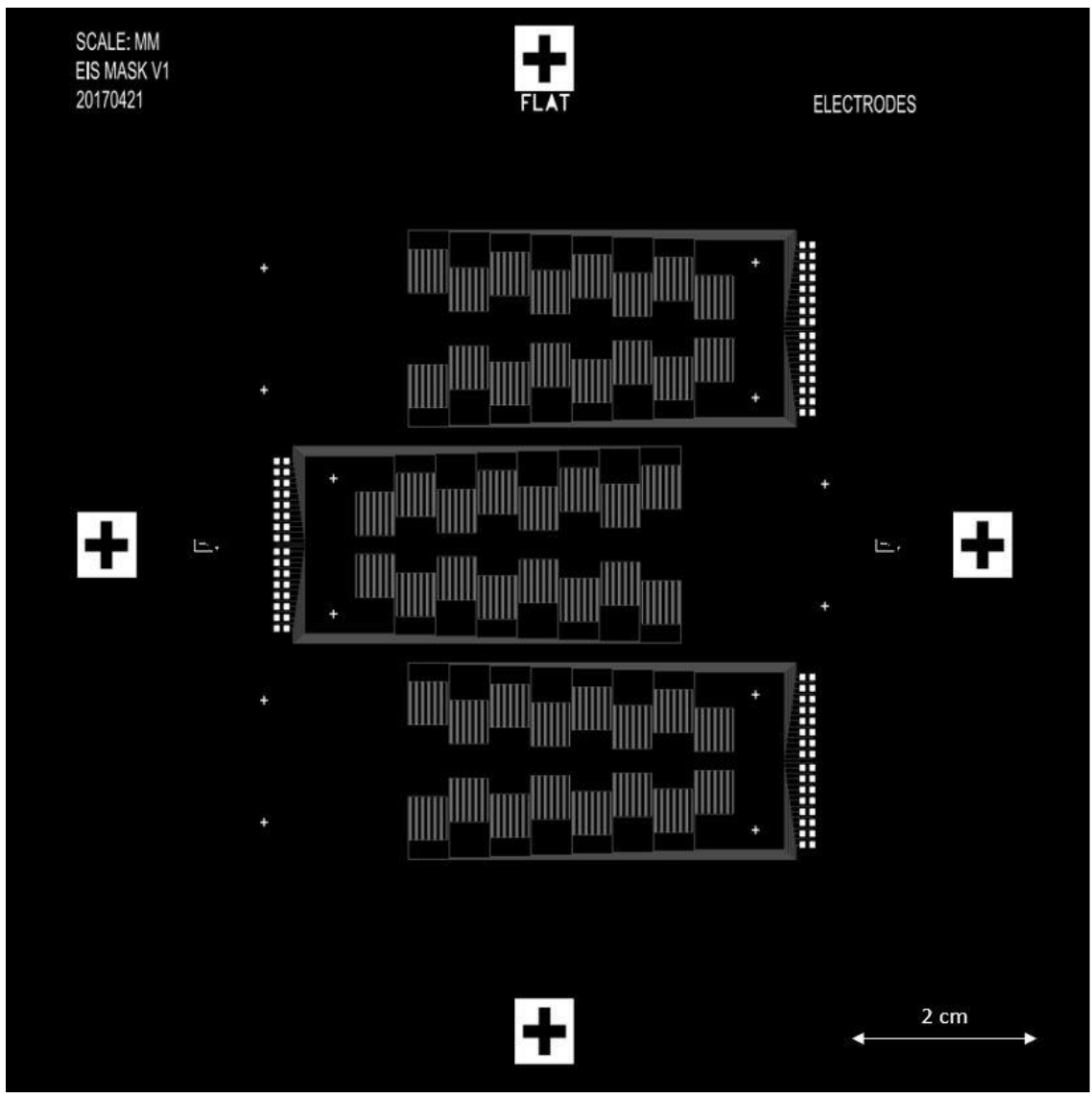

Figure 3.14 Electrode Array Photomask: Mylar photomask for Ma-N 1420 resist. Patterns the electrode array for lift-off.

\subsubsection{Metal Deposition}

With the patterned ma-N 1420 photoresist structure on the glass wafer, the next step is to deposit layers of metal onto the wafer's surface. These metal traces will form the electrode array for impedance measurements. A process known as physical vapor deposition (PVD) or sputtering is used, wherein ionized gas particles bombard a metal target, physically knocking metal off the target upon collision. These metal particles then adhere to the wafer's surface, forming a uniform metallic layer.

For the metal to properly adhere to the wafer, the wafer needs to be as clean as 


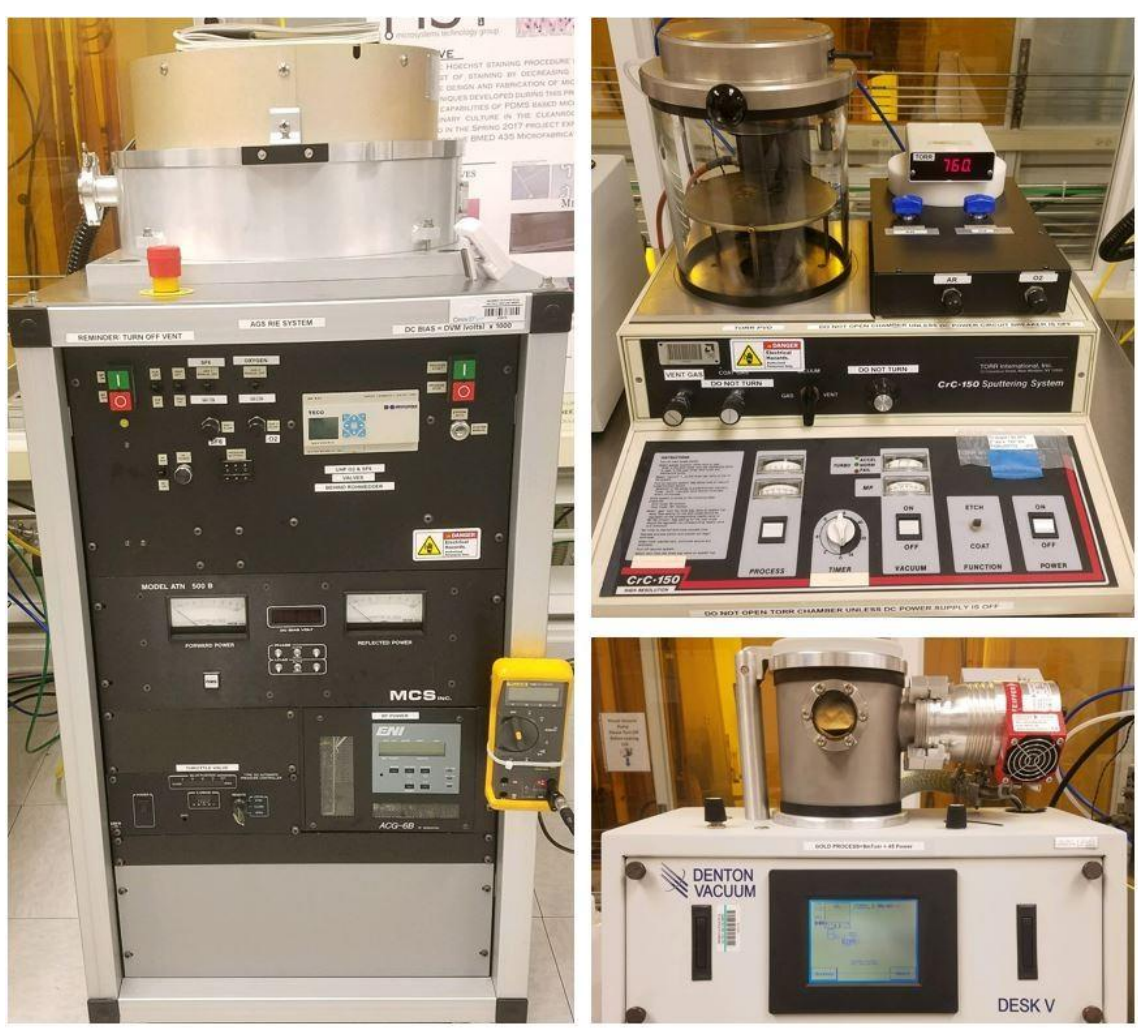

Figure 3.15 Machines for Physical Vapor Deposition: Left: Reactive Ion Etch machine. Top Right: Chrome sputter machine. Bottom Right: Gold sputter machine.

possible, free of any and all surface particulates and/or solvents. Previous chemical cleaning processes are unfavorable here, as the chemicals necessary to clean the wafer's surface would also damage the photoresist structure. Reactive ion etching (RIE) is used to clean the surface instead. Similar to PVD, gas particles are ionized and bombard the target, but instead of a metal target, the gas strikes the wafer's surface, removing unwanted surface material. While this may cause some aberrations in the resist, they are minor and do not compromise the array pattern. The wafer was placed in a specialized RIE chamber and oxygen gas was introduced at 300 mTorr. The oxygen was ionized for 30 seconds for cleaning the wafer surface and the chamber was vented. Machines used in this process are shown in Figure 3.15.

The first metal layer is a thin layer of chrome onto the wafer's surface. While the electrodes used for impedance spectroscopy were designed to be gold, gold does 
not adhere to the $\mathrm{SiO}_{2}$ wafer, so this initial chrome layer serves as an adhesive layer between the gold and glass wafer. The wafer was placed in a sputtering chamber with a chrome target and pumped down to 7 mTorr. Argon was introduced into the chamber, and was pumped down to 20 mTorr. The chamber was primed for 30 seconds and chrome was sputtered for 30 seconds, forming the chrome adhesion layer onto the wafer's surface.

Thewafer wasthen transferred to a separate sputtering machine containing agold target. Gold was sputtered onto the surfacefor 600 seconds, in two sets of 300 seconds each. This was done to minimize thermal generation during sputtering, potentially damaging the resist structure.

After sputtering the gold electrodes, another thin layer of chrome was sputtered over the gold. This layer follows the same process as the first chrome layer, but was only sputtered for 10 seconds instead of 30 seconds. This layer serves as a thin protective layer for the primary gold electrodes.

Microposit remover 1165, a resist stripper by MicroChem, was used to strip away the Ma-N 1420 photoresist from the wafer. This process will leave only metal on the surface of the wafer not covered by resist, forming the metal traces as desired. The wafer was immersed in the stripper at $70^{\circ} \mathrm{C}$, shown in Figure 3.16, along with a magnetic spinner to keep the acid continuously agitated, aiding to reduce the time taken to remove the resist. Heating elements for the remover were turned off overnight, increasing the time taken for resist removal but adhering to safety protocols. Tocheck for complete resist removal, the wafer was removed from the 1165 stripper, rinsed with deionized water, and immersed in isopropyl alcohol and/or gently wiped with a lint-free wipe dipped in isopropyl alcohol. If excess metals were still present on the surface, the wafer was immersed back into the remover. Upon completion the wafer was rinsed with deionized water, then spin-rinse-dried. 


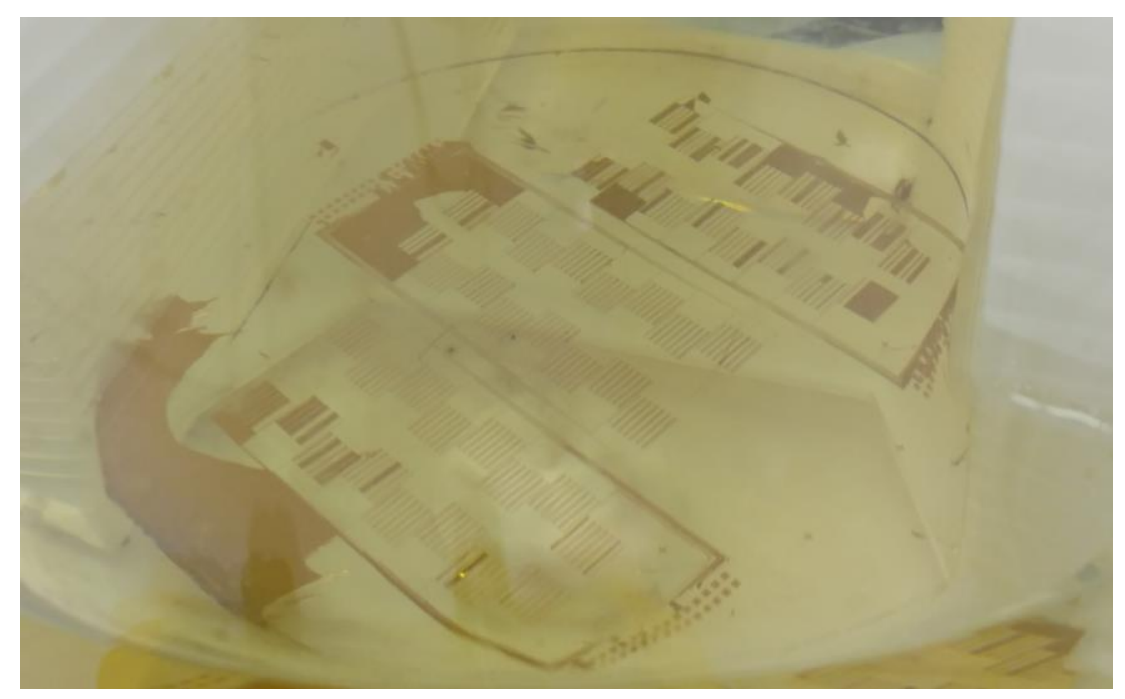

Figure 3.16 Immersion in Photoresist Strip: Electrode array immersed in photoresist stripper, leaving only metal traces on the wafer's surface.

Each of the three electrode arrays was then cut out of the wafer using a diamond blade dicing saw and cleaned with isopropyl alcohol.

\subsection{PDMS and Plasma Bonding}

PDMS was made using 30 grams of part A and 3 grams of part B per wafer poured. Contents were thoroughly mixed for several minutes and placed in a vacuum chamber to remove air introduced during mixing. Once all air was removed from the

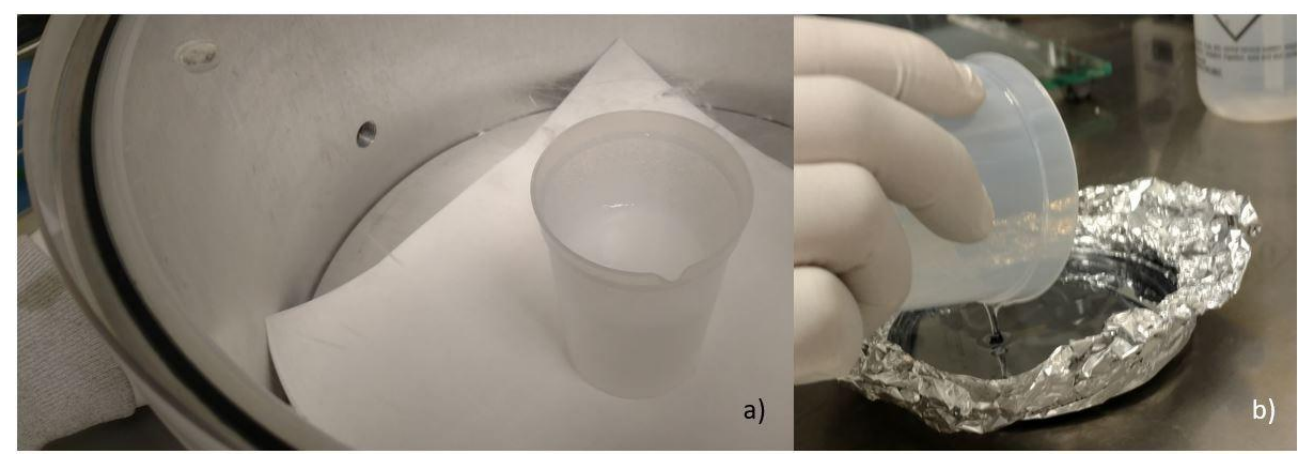

Figure 3.17 PDMS Processing: a) PDMS mixture under vacuum. b) PDMS pour onto the SU-8 coated wafer.

mixture, PDMS was poured evenly across the wafer, shown in Figure 3.17. Any air 
bubbles present after pouring were removed using tweezers. The wafer was placed in an oven at $70^{\circ} \mathrm{C}$ and PDMS was allowed to cure overnight. Do not use a plastic petri dish to house the wafer, as it will melt during the curing process. Glassware or aluminum foil was used to house the wafer.

The PDMS was removed from the oven and passively cooled to room temp, where a razorblade was used to cut PDMS around the wafer's edge and between the devices. It's important to ensure the blade has completely gone through the PDMS, else the PDMS will tear during removal process. PDMS was carefully peeled from the wafer's surface and placed "channel side up" (the side previously against the wafer). Tapecan be placed over the channels to store for a later date if needed. A $2 \mathrm{~mm}$ diameter punch was used to remove PDMS in the device inlet and outlet ports, using magnification to verify that the holes were cleanly punched.

The glass-electrode array was cleaned using isopropyl alcohol and low purity nitrogen gas. PDMS and electrodes were placed in a plasma cleaner. After pumping the system pressure down, the contents were exposed to air plasma for 15 seconds. The two were bonded together immediately afterward, ensuring any air in-between the two layers was removed. The wafer was placed in a $70^{\circ} \mathrm{C}$ oven overnight to aid in bond strength.

\subsubsection{Fluid Connection}

Bonded devices were plumbed with 14-gauge Tygon tubing attached to syringes with luer-lock blunt tip dispensing needles, and fluid was moved through the length of tubing. The tubing was then connected to the device inlet ports, shown by Figure

3.18. Another section of tubing was connected to the device outlet port and a waste cup. The order of tubing connections is important, as it minimizes unwanted air in the device, as can be seen in Figure 3.18 as well. The syringes were secured within the 


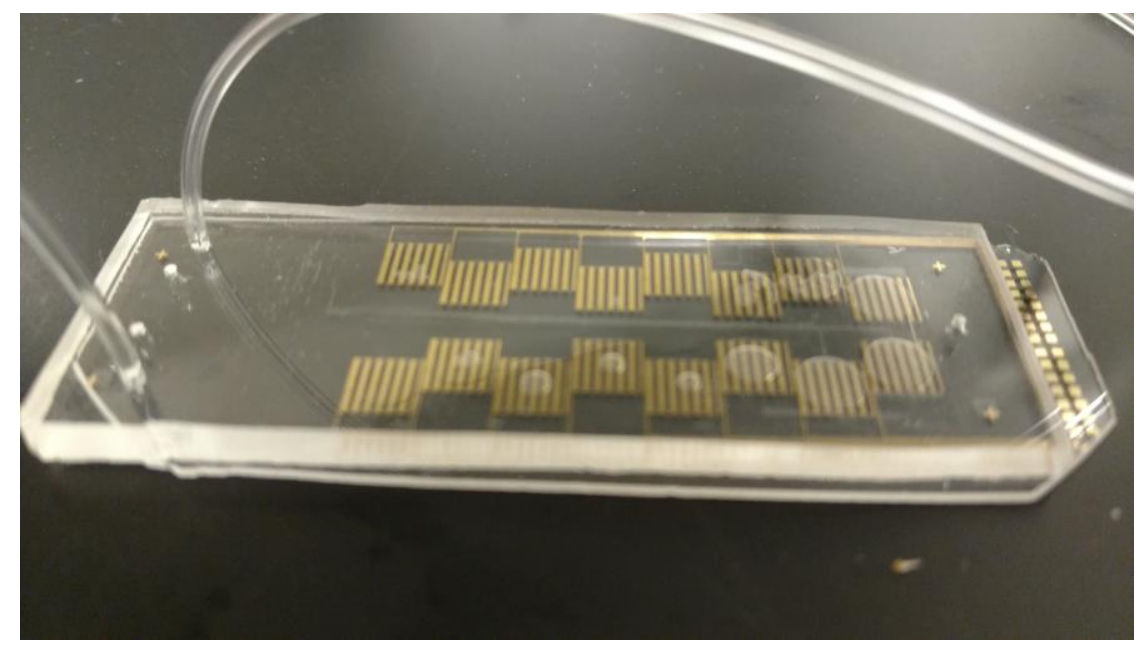

Figure 3.18 Tygon Tubing Connections: Tygon tubing connected to the cell seeding network inlets

syringe pump clamp and both pumps were set to the appropriate syringe diameter and flow rates.

\subsection{Safety Concerns}

Piranha and Buffered Oxide Etch are powerful acids. Photoresist stripper 1165 and Photoresist developers CD-26 and Ma-D 533/S are strong bases. Usage of these chemicals must be performed under a vented chemical hood with proper face, hand, and clothing protection. Avoid all skin contact with chemicals. Improper operation of UV exposure can cause eye damage. Use necessary shielding and avoid eye contact while machine is active. Gases used during the manufacturing process are under high pressure and various heating elements are used as well. Do not leave machines running unattended. Cutting the electrode arrays out of the glass wafer using a dicing poses several hazards. Use caution while operating a dicing saw and wear appropriate protection to avoid glass shards. 


\subsection{Device Data Collection}

\subsubsection{Instrumentation}

\section{Microscopy}

All microscopy measurements were taken using a SVM340 microscope by LabSmith. Using a 4x objective lens, image dimensions taken were 1.5 x $1.5 \mathrm{~mm}$. Three images were taken per well chamber and relevant values were averaged.

\section{Impedance}

From the representative circuit shown by Figure 2.3, two microactuator probes were connected as ocilloscopes 1 and 2, with the connecting nodes being the horizontal pair of bond pads on the device. This places the array of interdigitated electrodes as the device-under-test (DUT) in Figure 2.3. Nodes were connected via alligator clips, and the $100 \mathrm{k} \Omega$ resistor was grounded via breadboard. Digilent's Analog Discovery 2 was used for the voltage source, frequency sweep, oscilloscope channels, and grounding channel. The Analog Discovery 2 was connected to a PC workstation via microUSB. A LabView VI was written to conduct the frequency sweep, impedance, and phase changemeasurements. The code was setup to take measurements either along a userspecified time interval or as a one-time manual measurement. All frequency sweeps were conducted between $1000 \mathrm{~Hz}$ and $10 \mathrm{MHz}$. Probe contact to the bond pads was facilitated via a handheld 2x objective brightfield USB microscope.

\subsubsection{Gradient Generator}

The first set of fluidic runs tested the effectiveness of the concentration gradient. Yellow food dye was connected to one gradient inlet port, and blue food dye to the other. Both syringe pumps were set to a flow rate of $0.5 \mathrm{~mL} / \mathrm{hr}$. The second gradient 
test was conducted using $0.234 \%$ weight fluorescein in water in one inlet and pure water in the other inlet. Pumps were again set to $0.5 \mathrm{~mL} / \mathrm{hr}$. Per cell chamber, three images were taken and pixel intensity values were averaged.

\subsubsection{Ethanol Control}

The second fluidic run was to test the consistency of the impedance measurement setup. This test helps to verify that aberrant spikes in impedance do not occur during measurement, either due to errors in microactuator probe positioning, LabView errors, or unforeseen experimental factors. For this, $70 \%$ ethanol in water was run through the devicefor 1 hour at $0.5 \mathrm{~mL} / \mathrm{hr}$ with frequency sweep impedancemeasurements every 5 minutes. $70 \%$ ethanol was used here as it is used for device sterilization as serves as an excellent baseline for this control experiment.

\subsubsection{Saltwater}

The third fluidic run was to test the ability of the device/measurement apparatus to detect changes in impedance. For this, $1 \%$ weight $\mathrm{NaCl}$ in water was flown through the device for 10 minutes at $0.5 \mathrm{~mL} / \mathrm{hr}$, with impedance measurements every minute. Pure water was then flown through the device for 10 minutes to flush the system, then another 10 minutes with measurements again taken every minute.

\subsubsection{Cell Injection}

The fourth fluidic run was to study the effects of the presence of cells on the system's impedance. For cells, NIH-3T3 fibroblasts were cultured by Dr. Kristen Cardinal's lab at California Polytechnic University at San Luis Obispo. This cell line was chosen as they are naturally adhesive, robust, and readily available. Typically

growing $20 \mu \mathrm{m}$ in size and lying flat ( $\sim 1 \mu \mathrm{m}$ in height) on the surface, this cell 
line was an excellent candidate for exploratory research experiments. 70\% ethanol in water was run overnight at $0.5 \mathrm{~mL} / \mathrm{hr}$ to sterilize the device, with impedance measurements taken every hour. Then cell culture media, a solution of $500 \mathrm{~mL}$ DMEM high glucose, $50 \mathrm{~mL}$ FBS, $5 \mathrm{~mL} \mathrm{P} / \mathrm{S}$, and $0.6 \mathrm{~mL}$ Fungizone, was run through the device for 3 hours at $0.5 \mathrm{~mL} / \mathrm{hr}$ to flush the ethanol. Impedance measurements of the media every minute 10 minutes prior to cell injection. NIH-3 33 fibroblast cells were typsinized and injected into the cell seeding network at $1 \mathrm{~mL} / \mathrm{hr}$ at a concentration of $2.42 \times 10^{6}$ cells $/ \mathrm{mL}$. The increased flow rate for cell injection was used to avoid as much cell clumping as possible (as cells begin re-adhesion approximately 10 minutes post-typsinization). Impedance measurements were taken every minute for 5 minutes, the pumps were then turned off and impedance measurements were taken every 20 seconds for 2 minutes. Impedance measurements continued to be taken every minute for another 10 minutes. 


\section{Chapter 4}

\section{RESULTS/DISCUSSION}

\subsection{Manufacturing: SU-8 Mold}

\subsubsection{Alignment}

Originally, AZ-1529 was used to create the alignment marks themselves. The wafer was fully cleaned and no surface oxide was grown. During the SU-8 2007 spinon, however, theSU-8 had fully masked the thin AZ-1529 layer, causing the alignment structures to be unseen during pre-exposure. Without proper alignment, it was not possible to continue with the second SU-8 2050 layer, as channels and chambers would not be connected. To alleviate this, oxide was used instead to form the alignment structures, rather than the AZ-1529 photoresist. This caused the alignment marks to be visible through the SU-8 2007, allowing for subsequent layer alignment.

Even though the oxide allowed for visible alignment, marks were still very faint and difficult to observe. The microscope used for mask alignment to the wafer is an intrinsic component in the UV exposure machine, and was limited in its contrast ability. As such, even using the oxide alignment structures, one of the wafers had an error during the alignment during the SU-8 2050 exposure process. This error caused extreme overlap between the SU-8 2007 and SU-8 2050, rendering the entire wafer nonfunctional as delivery networks were not connected to cell chambers. As only two wafers were initially processed, this error on one of the final steps for the SU-8 mold resulted in an effective $50 \%$ loss in yield.

\subsubsection{Photoresist Spin}

Resist spinning was often unsuccessful. Adhesion issues of AZ-1529 were fairly consistent. More HMDS primer was used on the surface than originally planned to 
help alleviate this. Furthermore, SU-8 2007 films exhibited microbubbles and defects across the wafer during the spin process. These air bubbles remained after the softbake, and would not be acceptable to form the channel network. After the wafers were recleaned, more SU-8 2007 was poured onto the wafer's surface. This only proved to be marginally effective, and the wafers were cleaned again. Ensuring that the SU-8 2007 was at room temperature prior to spinning was the most effective method to reducetheseair bubbles. Every timetheresist was poorlyspun, acetoneand isopropyl alcohol were used to remove the resist, and the surface needed to be properly cleaned again, resulting in the wafer having to start back at step 1, significantly hindering processing time.

Furthermore, the spin-coat chamber needed to be lined with aluminum foil to prevent its side walls from being coated with photoresist. Every wafer processed needed to have the chamber's lid lifted to insert or remove the wafer. As more wafers were processed in a single session, excess photoresist would often fall off the foil and ontothewafer's surface. This resulted in the wafer needing to be cleaned and returned back to step 1, again severely hindering processing throughput.

\subsection{Manufacturing: Electrode Array}

\subsubsection{Photoresist Spin}

HMDS primer had numerous adhesion issues during spin coating. An example of these issues can be seen in Figure 4.1. This caused multiple defects within the Ma-N 1420 coat of photoresist, creating the need to reprocess the wafer. Wafers regularly needed to be reprocessed and still often had several minor adhesion issues. Using more resist and HMDS primer did help to create a more even coating as well as running low rpm spin cycles for longer to spread the resist.

In SU-8 photolithopgraphy, isopropyl alcohol (IPA) is used to rinse the wafer 
during the development process. IPAdissolves Ma-N 1420 photoresist, and was acci-

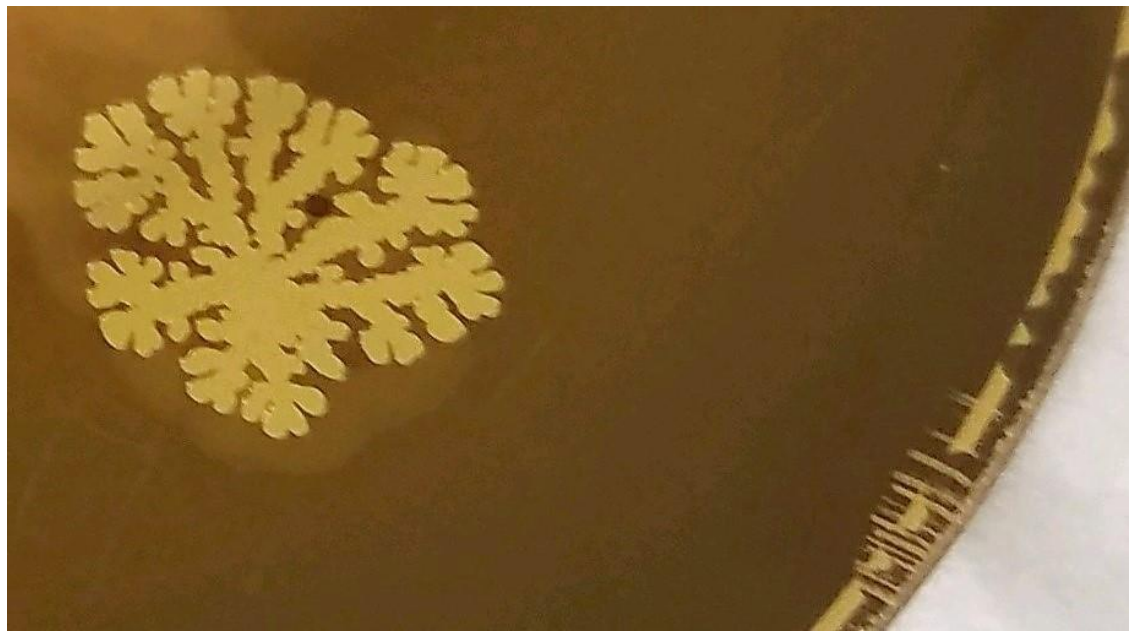

Figure 4.1 Sputtering Adhesion: HMDS primer adhesion issues during spin-coating, often indicated by the floral-like pattern

dentally used several times mistakenly during development. This resulted in having to fully reset multiple wafers, losing both time and laboratory resources.

\subsubsection{Sputtering}

There were two critical issues that occurred during metal deposition. The first was due to the nature of the sputtering process and the resources available. Chrome readily forms a native oxide on its surface. When the wafer was transferred from the chrome sputter to the gold sputter, the vacuum had to be broken, exposing the initial chromelayer to the atmosphere. While only seconds worth of atmospheric exposure, this chrome formed a very thin layer of oxide on its surface. While gold adheres to chrome, it does not adhere to chrome oxide. This causes the primary electrode metal, gold, to peel away from the chrome, either during resist removal or during experimentation. The second issue was due to human error. The resist spin and the metal deposition processes often took place on separate days, primarily due to the time required to setup and process. Glass wafers used in this are transparent, and the wafer was occasionally turned upside down during processing or transfer. The resist 
structure was too thin to know which side was right side up by visual inspection, or the flip simply went unnoticed. If the wafer did get accidentally flipped, metal would be deposited on the underside, and the wafer would need to be reprocessed from the beginning.
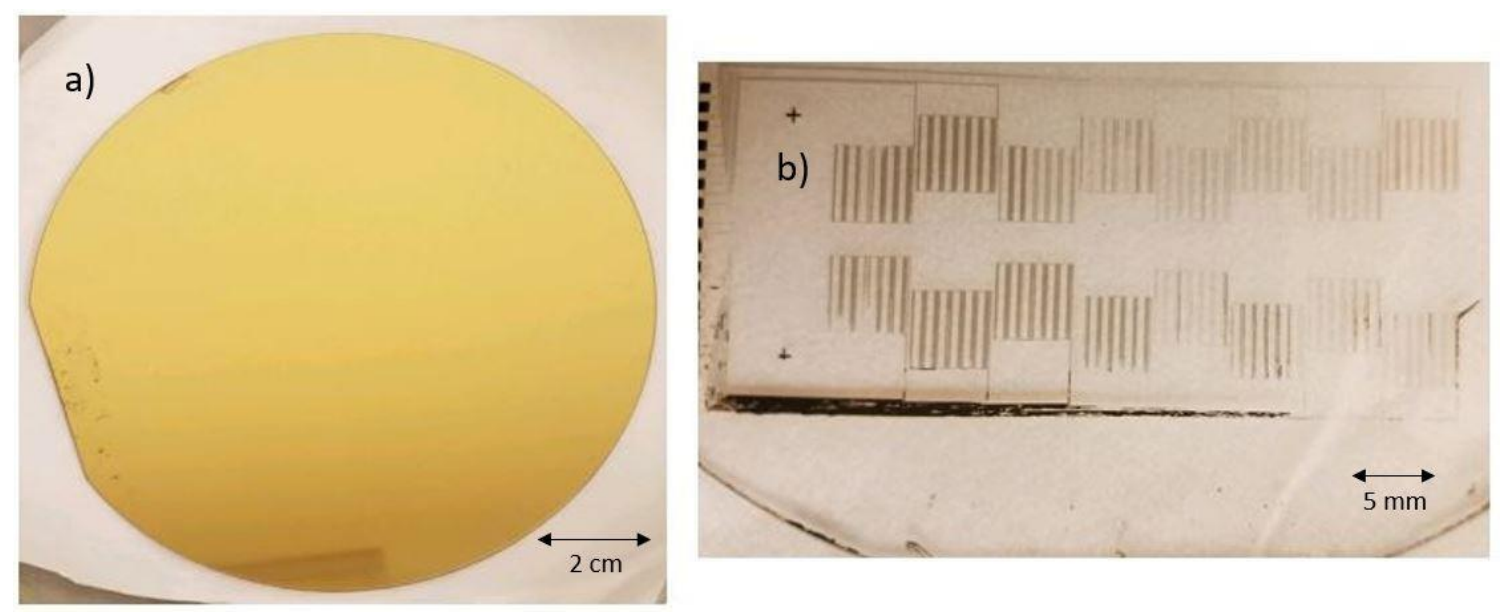

Figure 4.2 Sputtering Errors: Left: Gold sputter on the underside of the wafer. Right: Gold layer adhesion issues to initial chrome layer due to the presence of thin chrome oxide.

These issues, combined with other miscellaneous and unavoidable defects in the photoresist structure, caused several metal traces to have breaks in them, shorting the system circuit and rendering that cell chamber unusable for impedance data. Damage to the electrode array could also occur at high enough flow rates (>1.0 $\mathrm{ml} / \mathrm{hr}$ ), faulting the chamber. If a chamber was faulted, another was used for data collection, but with a low manufacturing yield and high electrode fault rate, very few chambers were capable of measuring impedance data. Keeping flow rates $<1.0 \mathrm{ml} / \mathrm{hr}$ helped to maintain electrode integrity.

\subsection{Characteristic Experiments}

Thefollowing set experiments were conducted to show gradient generation functionality and the device's overall ability to measure chamber impedance. These serve 
as preliminary trials to show further implications of cell culturing and dynamic analysis.

\subsubsection{Concentration Generator}

An initial COMSOL simulation was conducted to test the theoretical ability of the gradient generator channel network to create 16 varying concentrations. Concentration of dilute species COMSOL module was used for the study, with aqueous solution fluid properties and fluorescein diffusivity $\left(4.25 * 10^{-6} \mathrm{~cm}^{2} / \mathrm{s}\right)$. A $2 \mathrm{D}$ approximation was used in the study, with channel depths of 10 microns. As can be seen

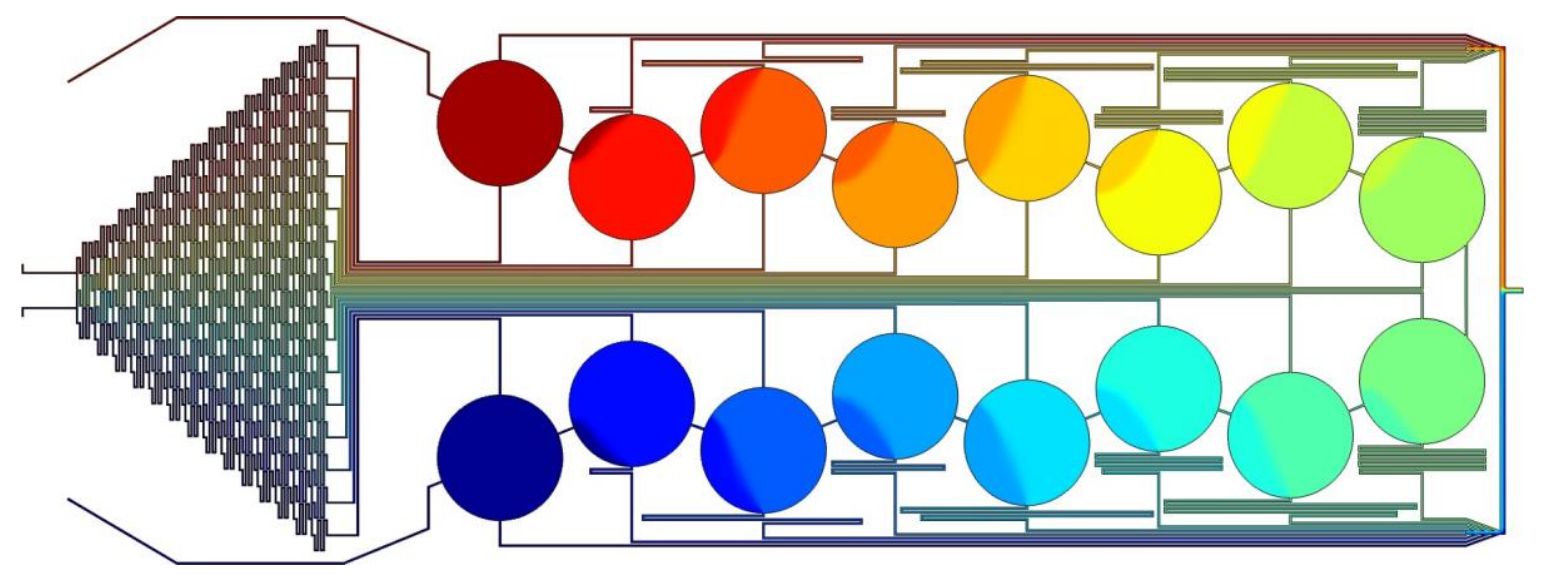

Figure 4.3 COMSOL Concentration Simulation: COMSOL Concentration Simulation. "Red" represents high solute concentration, whereas "Blue" represents low solute concentration.

in Figure 4.3, where red regions are the highest concentrations of solute, and blue regions are the lowest, the gradient generator does deliver varying concentrations to each culture well. There is some cross-chamber "contamination" due to the presence of the cell seeding network and its creation of an alternate path for fluid to travel. However, given the device's current design and the available resources, this is unavoidable. While a pneumatic valve system could be put in place to fix the cross-chamber, overall device complexities and local resources make this addition unfeasible at the present. 
The second test used yellow food dye in one gradient inlet and blue food dye in the other inlet. This test was used as a preliminary analysis of the gradient generator, showing mostly a "proof-of-concept" through visual color representation. Flow rates were set to $0.5 \mathrm{~mL} / \mathrm{hr}$ and allowed to run for at least 20 minutes to allow the gradient to form. Shown in Figure 4.4, blue, yellow, and green color channels were analyzed to determine each chamber's overall "color".

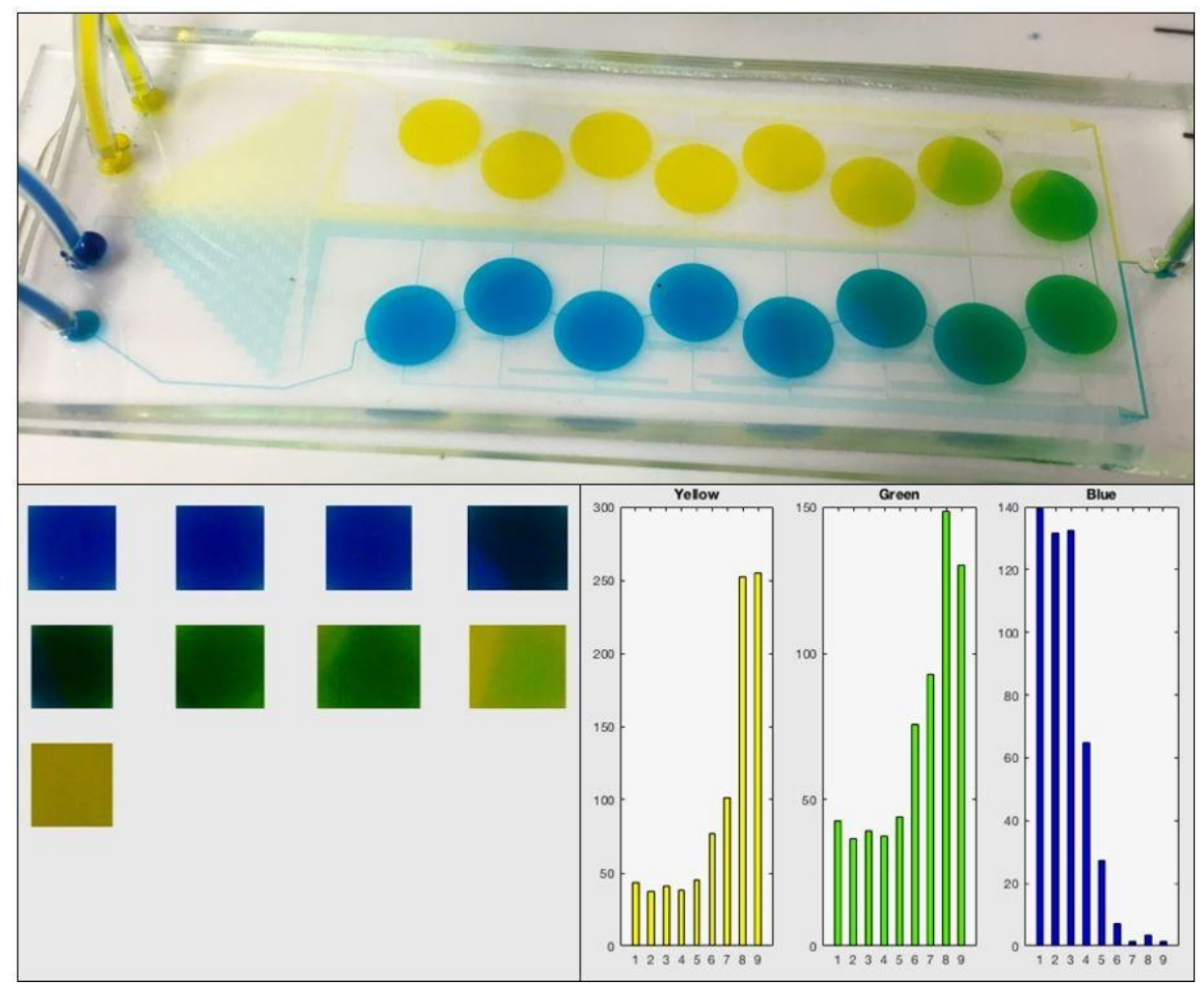

Figure 4.4 Food Dye Experiment: Food dye concentration experiment. Top: Device in use with colored dyes. Left: Images of wells taken through the SVM microscope. Right: Color channel pixel density (x-axis is well number, starting from bottom-left of the device.

The third test used $0.234 \%$ weight fluorescein in water. This test was used to further work with the SVM microscope through black and white modalities, along with an additional experiment with the gradient generator separating solute concentrations to the cell chamber's. Pixel density values were normalized to water, recorded, and graphed, shown in Figure 4.5. 


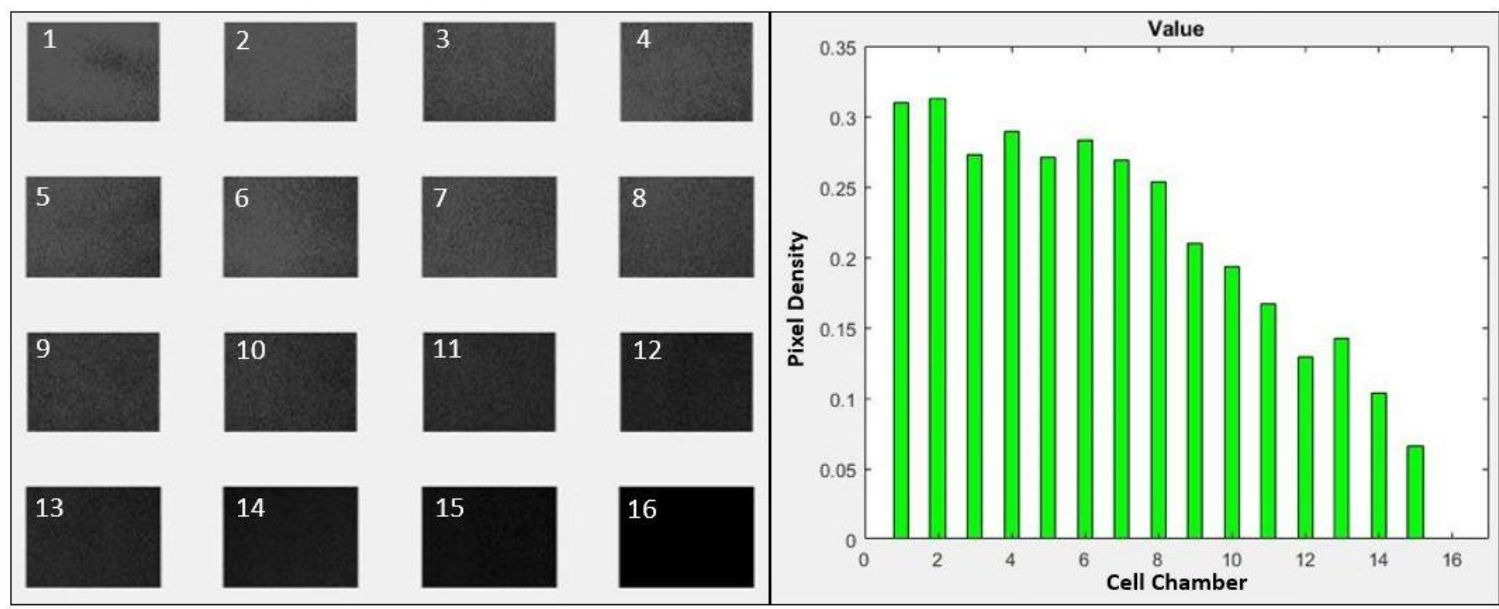

Figure 4.5 Fluorescein Concentration Experiment: Left: Images taken through SVM microscope. Right: Average pixel intensity per cell chamber. High pixel intensity = High fluorescein concentration

\subsubsection{Impedance Measurements}

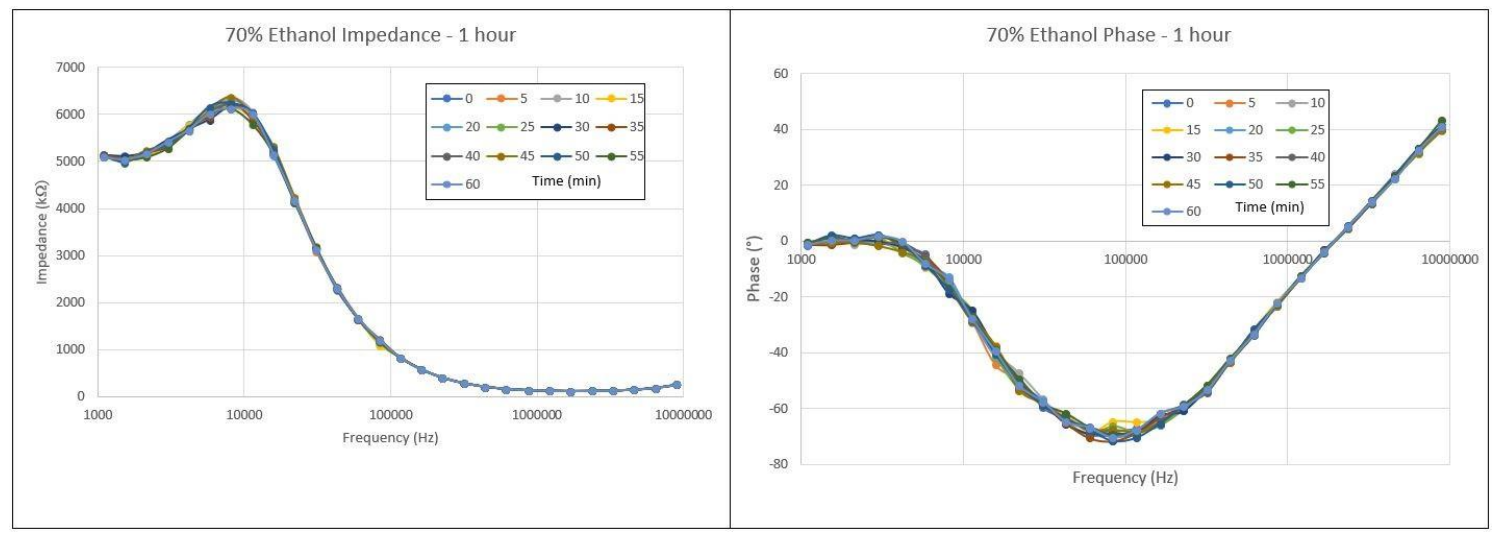

Figure 4.6 Ethanol Control Experiment: Left: Ethanol impedance plotted across frequencies for each time point. Right: Average phase measurement over time across frequencies. Note that since standard error was $<1 \%$ of value, error bars are not shown.

The ethanol control experiment was run to show that the device and circuit impedance measurement setup was capable of measuring phase and impedance in a consistent manner over time. It is important to note that over course of 1 hour of repeated measurements, no large changes in impedance were detected.

The saltwater experiment was conducted to see whether or not the device was 


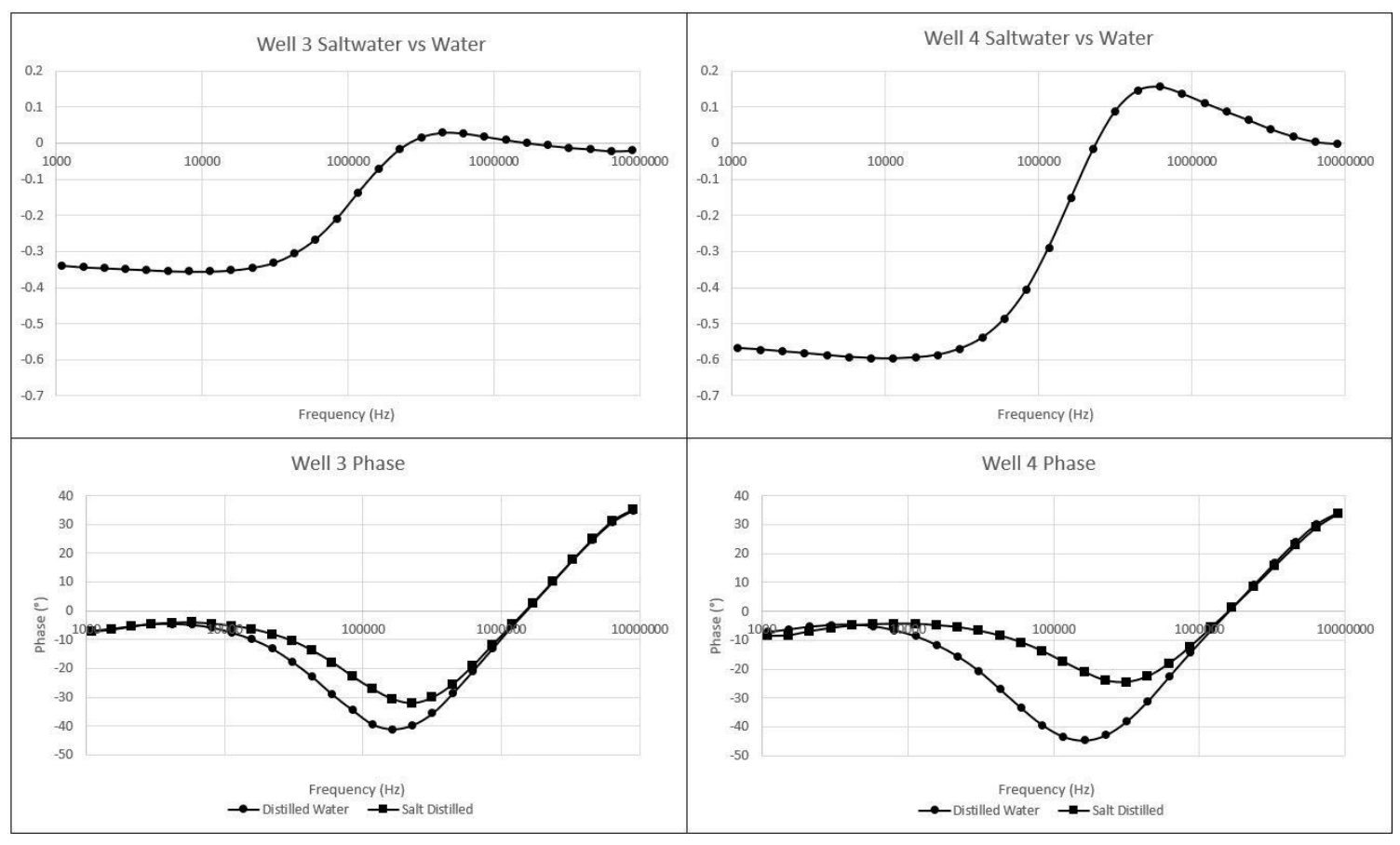

Figure 4.7 Saltwater impedance comparison experiment: Top: Relative impedance comparison between water and saltwater for 2 device chambers. Bottom: Phase measurements for both distilled water and saltwater fro 2 device chambers. Note that since standard error was $<1 \%$ of value, error bars are not shown.

able to switch fluids measure changes in impedance between fluids. Impedance measurements were compared between distilled water and $1 \%$ weight saltwater. Due to its higher conductivity, saltwater would be expected to havelower overall impedance, with differences most noticeable between $1000 \mathrm{~Hz}$ and $100 \mathrm{kHz}$ (figure 4.7), saltwater being $\sim 35 \%$ more conductive in chamber 3 and $\sim 60 \%$ more conductive in chamber 4. Above $100 \mathrm{kHz}$, the two fluid impedances were either indistinguishable or opposite of expected. This could be caused by parasitic elements within the system, or small changes in data collection (such as probe placement on the electrode pads). Phase measurements were also taken in this experiment to measure effects of capacitance and inductance present within the system.

$70 \%$ ethanol was run through the device to kill as much bacteria as possible. Ethanol measurements were taken, then cell media was run through the device, and 


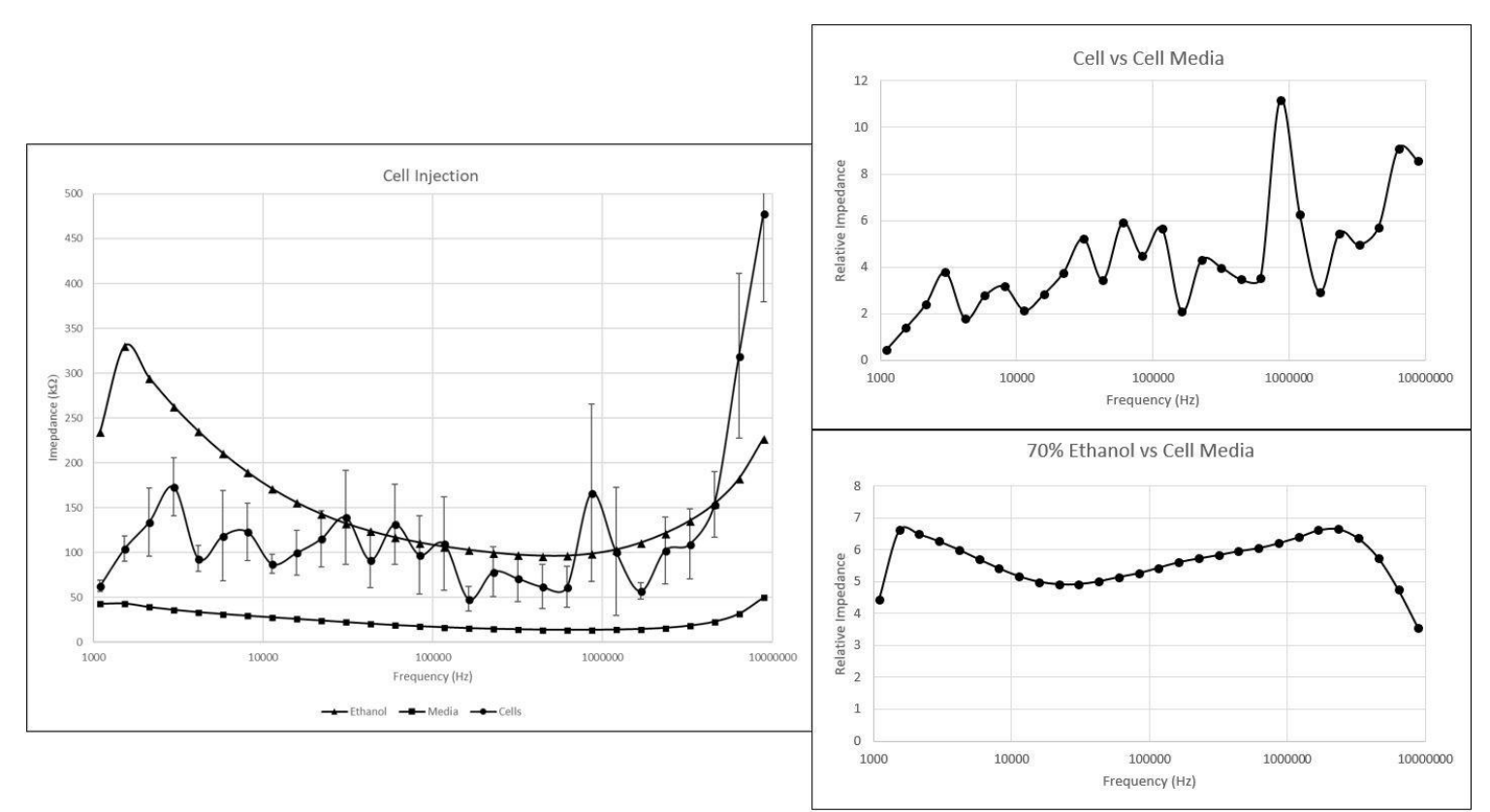

Figure 4.8 Cell injection experiment: Left: Impedance measurements comparing ethanol solution, cell media, and cells suspended in cell media. Right: Ethanol and cell measurements relative to cell media.

its measurements were taken. $3 \mathrm{~T} 3$ cells were then run through the device, and time intervals between measurements was shortened to capture as much data as possible. For 40 seconds, cell impedance measurements varied greatly at multiple frequencies. After 40 seconds, however, cell impedance readings returned back to the media baseline. Those 40 seconds of varying cell readings were averaged and graphed, shown in Figure 4.8. Graphs of cells and ethanol impedance against a media baseline are also included.

At the end of impedance measurements, $\mathrm{DiOC}_{6}$ dye was flown into the device in an attempt to image any cells in the device. Unfortunately, a high pressure build occurred, and blew out the PDMS-glass bond, ruining the device and causing leakage out the side of the device. The decision was made to avoid microscopy in this experiment to avoid potential damage to the microscope lens. 


\subsection{Complications}

The device, however, still has several complications. Gold's poor adhesion to the first chromelayer caused the electrodes to be fragile. Electrode arrays commonly had breaks or shorts in them. For the devices tested, even achieving two fully functioning electrode arrays proved difficult. Initially, the glass and PDMS were plasma treated for 30 seconds before being bonded together. Post-manufacturing, either during experimental setup of fluidic experimentation, the PDMS-glass bond often failed. This would either disrupt fluidic networks or cause fluid to flow out of the device, resulting in complete loss of functionality. Most bond failures occured at the inlet ports or along the PDMS edge. Manufacturing processes were also time-consuming and low-yield. Any minor photoresist defects, depending on location, could cause a full device failure. Errors in manufacturing, such as incorrect bake times, exposure, development, or spin-on often caused the need for a full wafer reset, losing hours of lab time and resources. This combined with device bond and electrode fragility caused only a few fluid experiments and data collection to be possible.

\subsubsection{Device Leaks}

Using syringe pumps to move fluid causes a very high pressure build at the inlet ports. Creating the inlet connection punch-hole during manufacturing could cause small damages in the PDMS. That, combined with an extended plasma treatment inevitably weakened the PDMS-glass bond leading to these failures. Normally, a high pressure build at the inlet port should simply eject the tygon tubing from the port, as the PDMS-glass bond should be stronger than the tubing-PDMS friction fit. Seeing bond failures here calls into question the strength of the bond over the rest of the device as well.

The device's PDMS edge walls were another common point of failure. This could 
be caused by geometric factors, that there is too little free space between the fluid channel and the metal traces. PDMS does not bond as strongly to these metal traces, potentially resulting in failure during a high pressure flow. Lowering the device footprint should help to alleviate this. Furthermore, a weak PDMS-glass bond caused other failures in various channel networks, disrupting planned fluid flow paths and device functionality.

At the end of impedance measurements, DiOC $_{6}$ dye flowed into the device in an attempt to image any cells in the device. Unfortunately, a high pressure build occurred, and blew out the PDMS-glass bond, ruining the device and causing leakage out the side of the device. The decision was made to avoid microscopy in this experiment to avoid potential damage to the microscope lens. If images were to be taken, it would be expected to find no cells present in the analysis chamber as impedance measurements returned to the media baseline shortly after injection. 


\section{Chapter 5 CONCLUSIONS}

The device was successfully able to generate multiple concentrations of fluid. The characteristic circuit setup successfully measured a chamber's impedance and phase changes across multiple frequencies over time. The device was able to distinguish changes in impedance between fluids, which was then utilized by introducing cells into the device, showing a change in impedance in the presence of biologics.

The lithography processes used were able to create a multi-layer structure of photoresist on a silicon wafer. This was used as a negative mold for PDMS, where the PDMS was casted, cured, and removed from the wafer, creating the series of fluidic networks throughout the device. An interdigitated electrode array of chrome-goldchrome was successfully patterned onto a glass wafer using lithography and physical vapor deposition. The photoresist was then chemically removed using a "lift-off" technique and the three arrays were then cut from the wafer.

The PDMS section containing the fluidic network and the glass containing the electrodes were then bonded together via air plasma, with each array of electrodes aligning over each chamber well. Tygon tubing connected the PDMS inlet ports to syringe pumps, and microactuator probes connected the device's bond pads to an oscilloscope logic analyzer, by which necessary frequency sweeps and impedance measurements were recorded.

\subsection{Experiments}

The COMSOL simulation, dye experiment, and fluorescein experiment showed that the device is capable of producing various fluid mixtures to several cell chambers. Likely due to unequal pump flow, variations in fluid resistance, or saturation of dyes, the concentration gradient generated was not sixteen distinct values as initially intended, but differences can still be seen between chambers. 
The ethanol experiment showed that the current impedance setup is able to measure the system's impedance on a consistent basis. It is important to show that, if all conditions are kept constant over time, the measurements remain the same as well.

The saltwater experiment showed the device is able to measure changes in impedance for fluids. Ideally, both chambers should have measured similar reductions in impedance when saltwater was introduced, but this was not the case.

The cell experiment showed the device's ability to detect the presence of biologics. Ethanol impedance was measured, then dropped when cell media, a conductivefluid, was introduced. For a short time during cell injection, impedance changes on the system were observed, then returned to the cell media's impedance levels. Knowing that the device consistently measures impedance from the previous ethanol experiment, and that changes in impedance can be measured from the previous saltwater experiment, this cell experiment provides a foundation for the detection of the presence of biologics in the system through impedance readings.

\subsection{Design Considerations}

Cell chambers with a $5 \mathrm{~mm}$ diameter are fairly large for microscale analysis. Without the PDMS support posts, the chambers bowed in due to the weight of the PDMS. The main problem from the large chamber size is the overall surface area for analysis. The large area of electrodes reduces the device's sensitivity to small changes in impedance (such as the presence of a small number of cells). Reducing the chamber diameter and inscribing the electrodes (diagonal of the array is the same as well diameter, not the array side length) should greatly improve overall sensitivity.

While the device does fit on a $25 \mathrm{~mm} \times 75 \mathrm{~mm}$ glass slide, it does not leave enough 
margin along the side walls. Low surface contact of the PDMS at critical stress points increases the chance of bond failure and device leakage, creating an alternate low resistance path for fluid flow. In a high pressure system, this path diverts nearly all flow through the failure point. Reducing the device's overall footprint, especially width-wise, should help reduce this chance of failure.

With multiple stages of fluid mixing to generate the concentration gradient, the fluid delivery network is quite long and requires a large amount of pressure for appreciable fluid flow. This high pressure system puts a large strain on the device, increasing chance of failure. To lower the system's overall pressure, lowering flow rates, increasing channel depth from $10 \mu \mathrm{m}$ to 15 or $20 \mu \mathrm{m}$, and lowering each characteristic mixer length could be utilized.

The micromanipulator probes used contained long wiring. Nodes were connected via alligator clips and the circuit was grounded via solderless breadboard. These connections typically exhibit high amounts of parasitics into the circuit (namely inductance via wiring and capacitance via breadboard). These parasitic elements lower the device's sensitivity, as they can mask changes in impedance caused bydifferent analytes. While parasitic electronics are present in every circuit, they can be minimized. Using a push-pin connection to the device pads and printed circuit board should help reduce these parasitic elements and aid in the measurement and connection simplicity.

\subsection{Future Work}

Devices need to have a higher manufacturing yield. Due to available resources, processing time were quite long, often several full work days for a single set of devices. Using more photoresist and ensuring the resist was at room temperature greatly improved spin-on success SU-8. It is important to follow all manufacturing processes carefully (such as correct bake times, not rinsing ma-D 533/S developer with IPA, and proper wafer handling). Simple errors, especially late in the manufacturing pro- 
cess, often required a full reset, resulting in multiple hours of lost work and wasted resources.

All devices should be plasma bonded for at most 15 seconds, not the initial 30 seconds. This extended oxidation caused the PDMS-glass bond to become tooweakto withstand the device's pressure requirements. During metal sputtering, the vacuum seal had to be broken between chrome and gold deposition, causing adhesion issues between the layers. Instead, silver should be used as a standalone metal layer, asit adheres to glass, avoiding the chrome oxide adhesion issue entirely.

The impedance measurement setup needs to be more streamlined. The microactuator probes and microscope-aided contact used were unwieldy. The probes had to be removed from the bond pads anytime tygon tubes needed to be switched or the device needed to be moved. Minor slips caused surface scratches, sometimes damaging the metal traces near the bond pads. To alleviate this and make taking impedance measurements more user-friendly, a pogo push-pin could be connected to a printed circuit board. Oscilloscope probes from the Analog Discovery 2 would then simply be connected to the PCB, and the device would be clamped in place to an external holder. This proposed connection should also reduce parasitic elements within the circuit as well.

Reducing chamber size will prevent PDMS bowing and inscribing the electrode array will increase the device's ability to detect impedance variations due to a decreased surface area for analysis. Using a more viscous photoresist than SU-8 2007 or lowering the spin speed will increase this layer's thickness and lower the overall pressure requirements of the system, reducing chance of bond failure. Using chrome-silver instead of chrome-gold for the electrode traces will increase overall yield. With these aforementioned changes and increased manufacturing yield, further experiments will be carried out to test the effects of fluid concentrations and appropriate cell responses through changes in impedance. 


\section{BIBLIOGRAPHY}

[1]Ma-N 400 and Ma-N 1400 - Negative Tone Photoresists. http://microchem.com/products/images/uploads/PI maN4001400.pdf. Accessed: 2018-07-03.

[2]A. R. Abate, D. Lee, T. Do, C. Holtze, and D. A. Weitz. Glass coating for pdms microfluidic channels by sol-gel methods. Lab Chip, 8:516-518, 2008.

[3]H. E. Ayliffe, A. B. Frazier, and R. D. Rabbitt. Electric impedance spectroscopy using microchannels with integrated metal electrodes. Journal of Microelectromechanical Systems, 8(1):50-56, 1999.

[4]J. Berthier and P. Silberzan. Microfluidics for biotechnology. Artech House, 2012.

[5]B. G. Chung, L. A. Flanagan, S. W. Rhee, P. H. Schwartz, A. P. Lee, E. S. Monuki, and N. L. Jeon. Human neural stem cell growth and differentiation in a gradient-generating microfluidic device. Lab on a Chip, 5(4):401, 2005.

[6]W. Commons. Cell membrane, 2018. [Online; accessed August 27, 2018].

[7]W.Commons. Doublelayer(surface sciene), 2018. [Online; accessed August $27,2018]$.

[8]W. Commons. Hodgkin-huxley model, 2018. [Online; accessed August 27, 2018].

[9]W. Commons. Laminar flow, 2018. [Online; accessed August 27, 2018].

[10]S. K. Dertinger, D. T. Chiu, Noo Li Jeon, and G. M. Whitesides. Generation of 
gradients having complex shapes using microfluidic networks. Analytical Chemistry, 73(6):1240-1246, 2001.

[11]R. Ehret, W. Baumann, M. Brischwein, Schwinde, B. Wolf, and K. Stegbauer. Monitoring of cellular behaviour interdigitated AG Medizinische impedance measurements on electrode structures. Biosensors \& Bioelectronics, 12:29-41, 1997.

[12]D. L. Englert, M. D. Manson, and A. Jayaraman. Flow-based microfluidic device for quantifying bacterial chemotaxis in stable, competing gradients. Applied and Environmental Microbiology, 75(13):4557-4564, 2009.

[13]S. Gawad, L. Schild, and P. H. Renaud. Micromachined impedance spectroscopy flow cytometer for cell analysis and particle sizing. Lab on a chip, 1:76-82, 2001.

[14]I. Giaever and C. R. Keese. Monitoring fibroblast behavior in tissue culture with an applied electric field. Proceedings of the National Academy of Sciences of the United States of America, 81(12):3761-3764, 1984.

[15]A. T. Giannitsis. Microfabrication of biomedical lab-on-chip devices. A review. Estonian Journal of Engineering, 17(2):109, 2011.

[16]F. A. Gomez. Biological applications of microfluidics. Wiley-Interscience, 2008.

[17]D. W. Greve, X. Huang, D. Nguyen, and M. M. Domach. Modeling of impedance of cell-covered electrodes. Proceedings of IEEE Sensors 2003 IEEE Cat No03CH37498, 2:1358-1363, 2003.

[18]K. Hiroshi, S. Yasuyuki, and F. Teruo. Organ/body-on-a-chip based on microfluidic technology for drug discovery. Drug Metabolism and Pharmacokinetics, 33:43-48, 2018. 
[19]B. Huh, Dongeun Matthews, A. Mammoto, M. Montoya-Zavala, H. Y. Hsin, and D. Ingber. Reconstituting Organ-Level Lung Functions on a Chip. Science, 328:1662-1668, 2010.

[20]R. J, R. C, T. L, K. F, M. I, L. S, J. A, B. E, and T. M. Open Biology.

[21]K. F. Lei. Review on impedance detection of cellular responses in micro/nano environment. Micromachines, 5(1):1-12, 2014.

[22]M. R. K. A. K. A. C. A. U. P. K. K. E. W. D. B. S. R. K. L. G. G. Mark J. Powers, Karel Domansky. A microfabricated array bioreactor for perfused 3D liver culture. Biotechnology and Bioengineering, 78:257-269, 2002.

[23]A. Mata, A. J. Fleischman, and S. Roy. Fabrication of multi-layer SU-8 microstructures. Journal of Micromechanics and Microengineering, 16(2):276-284, 2006.

[24]J. C. McDonald and G. M. Whitesides. Poly(dimethylsiloxane) as a material for fabricating microfluidic devices. Accounts of Chemical Research, 35(7):491-499, 2002.

[25]M. Mehling and S. Tay. Microfluidic cell culture. Current Opinion in Biotechnology, 25:95-102, 2014.

[26]S. Musah, A. Mammoto, T. C. Ferrante, S. S. F. Jeanty, M. Hirano-Kobayashi, T. Mammoto, K. Roberts, S. Chung, R. Novak, M. Ingram, T. Didar, S. Koshy, J. Weaver, G. M. Church, and D. E. Ingber. Mature induced-pluripotent-stem-cell-derived human podocytes reconstitute kidney glomerular-capillary-wall function on a chip. 1:0069, 052017.

[27]K. Oh, K. Lee, and B. Ahn et al. Design of pressure-driven microfluidic networks using electric circuit analogy. Lab Chip, 12(3):515-545, 2012. 
[28]S. Park, Y. Zhang, S. Lin, T.-H. Wang, and S. Yang. Advances in microfluidic pcr for point-of-care infectious disease diagnostics. Biotechnology Advances, 29(6):830-839, 2011.

[29]S. M. Radke and E. C. Alocilja. Design and fabrication of a microimpedance biosensor for bacterial detection. IEEE Sensors Journal, 4(4):434-440, 2004.

[30]S. M. Radke and E. C. Alocilja. A high density microelectrode array biosensor for detection of E. coli O157:H7. Biosensors and Bioelectronics, 20(8 SPEC. ISS.):1662-1667, 2005 .

[31]J. Ruan, L. Wang, M. Xu, D. Cui, X. Zhou, and D. Liu. Fabrication of a microfluidic chip containing dam, weirs and gradient generator for studying cellular response to chemical modulation. Materials Science and Engineering C, 29(3):674-679, 2009.

[32]O. Solution and C. A. Measurements. Improved Adhesion/Bonding dueto Plasma Treatment of PDMS, 2018.

[33]S. Souf. Recent advances in diagnostic testing for viral infections. Bioscience Horizons: The International Journal of Student Research, 9:hzwo10, 2016.

[34]Z. Stojek. The electrical double layer and its structure. Electroanalytical methods: Guide to experiments and applications, pages 3-8, 2010.

[35]A. G. G. Toh, Z. P. Wang, C. Yang, and N. T. Nguyen. Engineering microfluidic concentration gradient generators for biological applications. Microfluidics and Nanofluidics, 16(1-2):1-18, 2014.

[36]G. M. Walker, J. Sai, A. Richmond, M. Stremler, C. Y. Chung, and J. P. Wikswo. Effects of flow and diffusion on chemotaxis studies in a microfabricated gradient generator. Lab on a Chip, 5(6):611, 2005. 
[37]G. M. Whitesides. The origins and the future of microfluidics. Nature, 442(7101):368-373, 2006.

[38]G. M. Whitesides and A. D. Stroock. Flexible Methods for Microfluidics. 42(2001):42-48, 2001.

[39]Y. N. Xia and G. M. Whitesides. Soft lithography. Annual Review Of Materials Science, 37(5):551-575, 1998.

[40]L. Yang, Y. Li, C. L. Griffis, and M. G. Johnson. Interdigitated microelectrode (IME) impedance sensor for the detection of viable Salmonella typhimurium. Biosensors and Bioelectronics, 19(10):1139-1147, 2004.

[41]S. Zeng, B. Li, X. Su, J. Qin, and B. Lin. Microvalve-actuated precise control of individual droplets in microfluidic devices. Lab Chip, 9:1340-1343, 2009. 